Dominik J. Wrazidlo

\title{
A Fundamental Class for Intersection Spaces of Depth One Witt Spaces
}

Received: 21 April 2019 / Accepted: 4 August 2020 / Published online: 11 August 2020

\begin{abstract}
By a theorem of Banagl-Chriestenson, intersection spaces of depth one pseudomanifolds exhibit generalized Poincaré duality of Betti numbers, provided that certain characteristic classes of the link bundles vanish. In this paper, we show that the middle-perversity intersection space of a depth one Witt space can be completed to a rational Poincaré duality space by means of a single cell attachment, provided that a certain rational Hurewicz homomorphism associated to the link bundles is surjective. Our approach continues previous work of Klimczak covering the case of isolated singularities with simply connected links. For every singular stratum, we show that our condition on the rational Hurewicz homomorphism implies that the Banagl-Chriestenson characteristic classes of the link bundle vanish. Moreover, using Sullivan minimal models, we show that the converse implication holds at least in the case that twice the dimension of the singular stratum is bounded by the dimension of the link. As an application, we compare the signature of our rational Poincaré duality space to the Goresky-MacPherson intersection homology signature of the given Witt space. We discuss our results for a class of Witt spaces having circles as their singular strata.
\end{abstract}

\section{Introduction}

The method of intersection spaces has been introduced by Banagl $[2,3]$ to provide a spatial perspective on Poincaré duality for singular spaces. Following Banagl's original idea, such a theory should assign to a given singular space $X$ a family of intersection spaces - namely, spaces $I^{\bar{p}} X$ parametrized by a so-called perversity function $\bar{p}$-in such a way that, when $X$ is a closed, oriented $n$-dimensional pseudomanifold, generalized Poincaré duality $\widetilde{H}_{*}\left(I^{\bar{p}} X ; \mathbb{Q}\right) \cong \widetilde{H}^{n-*}\left(I^{\bar{q}} X ; \mathbb{Q}\right)$ holds for the reduced singular (co)homology groups across complementary perversities $\bar{p}$ and $\bar{q}$. Recall that this generalized form of Poincaré duality involving perversity functions originates from the well-established intersection homology theory $I H_{*}^{\bar{p}}(X ; \mathbb{Q})$ of Goresky-MacPherson [18,19].

The purpose of this paper is to upgrade the middle perversity intersection space of a depth one Witt space to a rational Poincaré duality space. The fundamental class will be constructed by a single cell attachment in the top degree. Before

D. J. Wrazidlo ( $\varangle$ ): Institute of Mathematics for Industry, Kyushu University, 744 Motooka, Nishi-ku, Fukuoka 819-0395, Japan

e-mail: d-wrazidlo@imi.kyushu-u.ac.jp

Mathematics Subject Classification: 55N33 - 57P10 -55P62 -55R10 $55 \mathrm{R} 70$

https://doi.org/10.1007/s00229-020-01238-7 
discussing our results (see Sect. 2), which build on work of Klimczak [23] and Banagl-Chriestenson [7], we give in the following an outline of several existing results in the theory of intersection spaces.

In comparison with Banagl's intersection space homology theory $H I_{*}^{\bar{p}}(X ; \mathbb{Q})=$ $H_{*}\left(I^{\bar{p}} X ; \mathbb{Q}\right)$ one can observe that Goresky-MacPherson's intersection homology, and also Cheeger's $L^{2}$ cohomology of Riemannian pseudomanifolds [12-14], arise from certain intermediate algebraic chain complexes rather than from spatial modifications. The intersection space construction itself modifies a space only near its singular strata: Loosely speaking, each singularity link is replaced by a spatial approximation that truncates its homology in a degree dictated by the perversity function. Motivation for using such spatial homology truncations (Moore approximations) of the links comes from a similar behavior of intersection homology groups in the case of isolated singularities (see Section 2 in [2]). As it turns out, the homology theories $H I_{*}^{\bar{p}}$ and $I H_{*}^{\bar{p}}$ (as well as their corresponding cohomology theories) are in general not isomorphic. However, at least in the case of singular Calabi-Yau threefolds, they are related via mirror symmetry (see [3]).

Whenever intersection spaces exists, they serve as a source of desirable features that are not available in the context of intersection homology. For instance, intersection space cohomology comes automatically equipped with a perversity internal cup product. Moreover, addressing a problem suggested by Goresky and MacPherson in [20], intersection spaces provide an approach to construct generalized homology theories for singular spaces, like intersection $K$-theory (see Chapter 2.8 in [3], as well as [26]). Naturally, the advantages of the theory $H I_{*}^{\bar{p}}$ over $I H_{*}^{\bar{p}}$ come at the cost that the existence of intersection spaces which satisfy generalized Poincaré duality is far from granted. For pseudomanifolds with isolated singularities, intersection spaces do always exist, and their duality theory is well-studied [3]. However, for pseudomanifolds with more complicated singularities, the implementation of intersection space theory becomes rapidly more involved. This is already evident in the case of arbitrary two strata pseudomanifolds: Surprisingly, even if an intersection spaces can be constructed, the existence of a generalized Poincaré duality isomorphism turns out to be obstructed in general. As discovered by Banagl and Chriestenson [7], the failure of duality is precisely measured by local duality obstructions, which are certain characteristic classes associated to the link bundle over the singular stratum of the pseudomanifold. These obstruction classes are abstractly definable for fiberwise truncatable fiber bundles, and they vanish for product bundles and certain flat bundles, but not for generally twisted bundles. For some specific three strata pseudomanifolds with bottom stratum a point, a duality result for intersection spaces has been established in [4]. By developing an inductive method of intersection space pairs, Agustín and Fernández de Bobadilla have proposed in their recent preprint [1] a quite general construction of intersection spaces for pseudomanifolds of arbitrary stratification depth, at least when the link bundles can be compatibly trivialized. However, an obstruction theory for generalized Poincaré duality of intersection space pairs is not known (compare problem (6) in Section 2.6 in [1]). 
In view of the difficulties that arise in constructing intersection spaces, it seems beneficial to study intersection space homology by means of techniques that avoid constructing the intersection space itself. Notable alternative approaches are via $L^{2}$ theory [8], via linear algebra [17], via sheaf theory [1], and via differential forms $[5,15]$. The approach via $L^{2}$ theory applies to two strata pseudomanifolds having trivial link bundle. As for the linear algebra approach, Geske [17] constructs socalled algebraic intersection spaces on the chain level. His construction is based on a generalization of Moore approximations to multiple degrees that might in general not be realizable as a spatial modification of a tubular neighborhood of the singular set. While algebraic intersection spaces that satisfy generalized Poincaré duality exist for a large class of Whitney stratified pseudomanifolds, they are remote from the spatial concept in that they do not exhibit a natural cup product on cohomology, and the generalized local duality obstructions of Banagl-Chriestenson can be shown to vanish for an appropriate choice of the local intersection approximation (see Theorem 4.10 in [17]). On the level of homology, algebraic intersection spaces turn out to be non-isomorphic to the intersection space pair approach of Agustín and Fernández de Bobadilla (see Section 6 in [17]). Note that in [1], Agustín and Fernández de Bobadilla pursue a sheaf theoretic approach that is inspired by work of Banagl, Maxim and Budur [6,9,10,24]. Namely, they associate to intersection space pairs certain constructible sheaf complexes on the original pseudomanifold satisfying axioms analogous to those of the intersection chain complex in intersection homology theory [19]. Then, in Theorem 10.6 in [1] they show that so-called general intersection space complexes give rise to generalized Poincaré duality for two strata pseudomanifolds. Finally, concerning the differential form approach, we note the special and important feature that wedge product of forms followed by integration induces a canonical non-degenerate intersection pairing on cohomology in analogy with ordinary de Rham cohomology.

Returning to the original spatial approach, Klimczak [23] pursues the idea to realize Poincaré duality for intersection spaces by cup product followed by evaluation with a fundamental class rather than only showing equality of complementary Betti numbers. Let us consider the important case of a Witt space $X$ with isolated singularities. In this case, the intersection spaces associated to the lower middle and upper middle perversities $\bar{m}$ and $\bar{n}$ exist, and can be chosen to be equal, $I X=I^{\bar{m}} X=I^{\bar{n}} X$. By a Klimczak completion of $I X$ we shall mean a rational Poincaré duality space of the form $\widehat{I X}=I X \cup e^{n}$, where $n$ denotes the dimension of $X$. If $I X$ admits a Klimczak completion, then the fundamental class in $H_{n}(\widehat{I X})$ arises from the newly attached top-dimensional cell $e^{n}$, and an easy Mayer-Vietoris argument implies that the Betti numbers of $I X$ and $\widehat{I X}$ agree in degrees $1, \ldots, n-1$. In [23] it is shown that Klimczak completions exist for compact Witt spaces having isolated singularities with simply connected links. Moreover, the rational homotopy type of a Klimczak completion of a simply connected intersection space is determined by the intersection space according to a theorem of Stasheff [27] (see Remark 6.5). In view of future applications it seems interesting to invoke rational surgery theory to realize Klimczak completions by manifolds.

In this paper we study Klimczak completions for middle perversity intersection spaces of compact depth one Witt spaces. Future study will have to clarify 
the obstructions to the existence of Klimczak completions in the case of higher stratification depth.

The paper is structured as follows. Section 2 presents our main results in case of a two strata Witt space. In Sect. 3 we list some notation that will be used throughout the paper. Sections 4 and 5 contain the proofs of our main technical results. Finally, in Sect. 6, we prove our main results for depth one Witt spaces, and illustrate them in an example.

\section{Statement of results}

In this paper we study Klimczak completions for middle perversity intersection spaces of compact depth one Witt spaces. For this purpose, we adopt the framework of Thom-Mather stratified spaces as presented in Section 8 of [7]. For simplicity, we consider in the following a two strata Witt space $X$ with singular stratum $B$. Then, the Thom-Mather control data induce a possibly twisted smooth fiber bundle $E \rightarrow B$ with fiber the link $L$ of $B$ such that the complement of a suitable tubular neighborhood of $B$ in $X$ is a smooth $n$-manifold $M$ with boundary $\partial M=E$. In this setting, as explained in Section 10 of [7], the intersection spaces $I^{\bar{m}} X$ and $I^{\bar{n}} X$ associated to the lower middle and upper middle perversities $\bar{m}$ and $\bar{n}$ exist and can be chosen to be equal, $I X=I^{\bar{m}} X=I^{\bar{n}} X$, provided that the fiber $L$ admits an equivariant Moore approximation $f_{<}: L_{<} \rightarrow L$ of degree $\left\lfloor\frac{1}{2}(\operatorname{dim} L+1)\right\rfloor$ (with respect to a suitable structure group for $E \rightarrow B$ ). In view of Theorem 9.5 in [7] one might speculate that vanishing of Banagl-Chriestenson's local duality obstructions for $E \rightarrow B$ is in some way related to existence of a Klimczak completion for $I X$ because both assumptions imply Poincaré duality for the Betti numbers of $I X$. In this context, a central role is played by the truncation cone, cone $\left(F_{<}\right)$, the mapping cone of the fiberwise truncation $F_{<}: \mathrm{ft}_{<} E \rightarrow E$ induced by $f_{<}$. Namely, the local duality obstructions of the bundle $E \rightarrow B$ vanish if and only if all $(n-1)$-complementary cup products in $\widetilde{H}^{*}\left(\operatorname{cone}\left(F_{<}\right)\right)$vanish, where $n$ denotes the dimension of $X$. Moreover, when $B$ is a point and $L$ is simply connected, then the construction of the Klimczak completion for the intersection space $I X=$ cone $\left(F_{<}\right) \cup_{\partial M} M$ is implemented in [23] (see Section 3.2.1 and also Proposition 3.11 therein) as follows. In a first local step, an $n$-cell $e^{n}$ is attached to the truncation cone to produce a Poincaré duality pair $\left(\operatorname{cone}\left(F_{<}\right) \cup e^{n}, E\right)$, which is then in a second global step glued to the regular part $(M, \partial M)$ of $X$ to yield the desired rational Poincaré duality space $\widehat{I X}$. Generalizing to an arbitrary singular stratum $B$ and arbitrary link $L$, our Theorem 4.2 states that a Poincaré duality pair of the form (cone $\left(F_{<}\right) \cup e^{n}, E$ ) exists if and only if the rational Hurewicz homomorphism of the truncation cone in degree $n-1$,

$$
\operatorname{Hur}_{n-1 *}: \pi_{n-1}\left(\operatorname{cone}\left(F_{<}\right), \mathrm{pt}\right) \otimes_{\mathbb{Z}} \mathbb{Q} \rightarrow H_{n-1}\left(\operatorname{cone}\left(F_{<}\right)\right),
$$

is surjective. Moreover, we show that the local duality obstructions for $E \rightarrow B$ vanish necessarily in that case, which reveals some part of their homotopy theoretic nature. As a counterpart of Theorem 9.5 in [7], we show in Remark 6.4 that our Theorem 4.2 implies the following 
Theorem 2.1. Let $(X, B)$ be a compact two strata Witt space of dimension $n \geq 3$. Assume that the link $L$ admits an equivariant Moore approximation $f_{<}: L_{<} \rightarrow L$ of degree $\left\lfloor\frac{1}{2}(\operatorname{dim} L+1)\right\rfloor$. If the rational Hurewicz homomorphism of the associated truncation cone (see (1)) is surjective in degree $n-1$, then the middle perversity intersection space IX admits a Klimczak completion.

More generally, our method applies to depth one Witt spaces with more than one singular stratum (see Theorem 6.3(a)). Note that when $B$ is a point and $L$ is simply connected, we recover Klimczak's original result. Recall that the argument in [23] uses the rational Hurewicz theorem to show that the rational Hurewicz homomorphism of the truncation cone is always surjective in the relevant degree. We do not need to assume that the link is simply connected by employing the results of [28] for constructing Moore approximations for arbitrary path connected cell complexes.

According to Theorem 4.2, our surjectivity condition on the rational Hurewicz homomorphism is sufficient for the local duality obstructions to vanish. However, even in the case of a globally trivial link bundle, we do in general not know whether the converse implication is also true. Nevertheless, under the additional assumption that the truncation cone is simply connected, the converse implication can be analyzed further by means of minimal Sullivan models from rational homotopy theory (see Corollary 5.7 and Remark 5.8). In particular, in view of Theorem 4.2, an important consequence of our Theorem 5.1 is the following

Theorem 2.2. (see Remark 6.4) Let $(X, B)$ be a compact two strata Witt space of dimension $n \geq 3$. Assume that the link $L$ admits an equivariant Moore approximation $f_{<}: L_{<} \rightarrow L$ of degree $\left\lfloor\frac{1}{2}(\operatorname{dim} L+1)\right\rfloor$. Suppose that cone $\left(F_{<}\right)$, the associated truncation cone, is simply connected. If

$$
n<3 \cdot\left\lceil\frac{1}{2}(\operatorname{dim} L+1)\right\rceil= \begin{cases}\frac{3}{2}(\operatorname{dim} L+1), & \operatorname{dim} L \text { odd }, \\ \frac{3}{2}(\operatorname{dim} L+2), & \operatorname{dim} L \text { even },\end{cases}
$$

or, equivalently,

$$
\operatorname{dim} B=n-1-\operatorname{dim} L<\left\{\begin{array}{l}
\frac{1}{2}(\operatorname{dim} L+1), \quad \operatorname{dim} L \text { odd }, \\
\frac{1}{2}(\operatorname{dim} L+4), \quad \operatorname{dim} L \text { even, }
\end{array}\right.
$$

then the following statements are equivalent:

(i) The local duality obstructions of the link bundle vanish, that is, all (n-1)complementary cup products in $\widetilde{H}^{*}\left(\operatorname{cone}\left(F_{<}\right)\right)$vanish.

(ii) The rational Hurewicz homomorphism of the truncation cone (see (1)) is surjective in degree $n-1$.

The assumption that the truncation cone is simply connected is valid in many cases of practical interest - for instance, whenever the link has abelian fundamental group as pointed out in Example 4.18, or when the link bundle is trivial, see Example 4.19.

If the dimension of the Witt space $X$ is of the form $n=4 d$, then it is natural to study the symmetric intersection form $H_{2 d}(\widehat{I X}) \times H_{2 d}(\widehat{I X}) \rightarrow \mathbb{Q}$ of a Klimczak completion $\widehat{I X}$. In accordance with the results of Section 11 in [7], we can compare it to the Goresky-MacPherson-Siegel intersection form $I H_{2 d}(X) \times I H_{2 d}(X) \rightarrow \mathbb{Q}$ on middle-perversity intersection homology (see Section I.4.1 in [25]) as follows. 
Theorem 2.3. (see Remark 6.4) Let $(X, B)$ be a compact two strata Witt space of dimension $n=4 d$. Suppose that the rational Hurewicz homomorphism of the truncation cone (see (1)) is surjective in degree $n-1$. Then, the Witt element $w_{H I} \in$ $W(\mathbb{Q})$ induced by the symmetric intersection form $H_{2 d}(\widehat{I X}) \times H_{2 d}(\widehat{I X}) \rightarrow \mathbb{Q}$ of the Poincaré duality space $\widehat{I X}$ equals the Witt element $w_{I H} \in W(\mathbb{Q})$ induced by the Goresky-MacPherson-Siegel intersection form I $H_{2 d}(X) \times I H_{2 d}(X) \rightarrow \mathbb{Q}$ on middle-perversity intersection homology. In particular, it follows that the two intersection forms have equal signatures.

We point out that our proof of Theorem 6.3(c) exploits massively the existence of a fundamental class for $\widehat{I X}$, which enables us to invoke Novikov additivity for Poincaré duality pairs (see Lemma 3.4 in [23]). On the other hand, lacking the existence of a fundamental class under the assumption that the local duality obstructions vanish, the argument of Banagl-Chriestenson in Section 11 in [7] requires an involved construction of an abstract, non-canonical symmetric intersection form for $I X$. It seems to be an interesting problem to compare intersection forms of Klimczak completions to intersection forms that arise from the differential form approach [5].

In Sect. 6.2 we provide a class of examples of depth one Witt spaces with twisted link bundles for which our Theorem 6.3 applies. For further examples concerning the existence of equivariant Moore approximations in general we refer to Sections 3 and 12 in [7].

\section{General notation}

We collect some general notation that will be used throughout the paper.

By a pair of spaces $(X, A)$ we mean a topological space $X$ together with a subspace $A \subset X$. A pointed pair of spaces $\left(X, A, x_{0}\right)$ is a pair of spaces $(X, A)$ together with a basepoint $x_{0} \in A$. A map of pairs $f:(X, A) \rightarrow\left(X^{\prime}, A^{\prime}\right)$ is a map $f: X \rightarrow X^{\prime}$ such that $f(A) \subset A^{\prime}$.

Let $D^{p}=\left\{x \in \mathbb{R}^{p} ; x_{1}^{2}+\cdots+x_{p}^{2} \leq 1\right\}$ denote the closed unit ball in Euclidean $p$-space $\mathbb{R}^{p}$, and $S^{p-1}:=\partial D^{p}$ the standard $(p-1)$-sphere. We also fix $s_{0}=1 \in$ $S^{0} \subset S^{p-1}$ as a basepoint.

Given a pointed pair of spaces $\left(X, A, x_{0}\right)$, the Hurewicz map in degree $n \geq 1$ is

$$
\operatorname{Hur}_{n}: \pi_{n}\left(X, A, x_{0}\right) \rightarrow H_{n}(X, A ; \mathbb{Z}), \operatorname{Hur}_{n}([f])=f_{*}(\nu)
$$

where $f_{*}: H_{n}\left(D^{n}, \partial D^{n} ; \mathbb{Z}\right) \rightarrow H_{n}(X, A ; \mathbb{Z})$ is induced by a representative

$$
f:\left(D^{n}, \partial D^{n}, s_{0}\right) \rightarrow\left(X, A, x_{0}\right)
$$

of $[f] \in \pi_{n}\left(X, A, x_{0}\right)$, and $v$ denotes a fixed generator of $H_{n}\left(D^{n}, \partial D^{n} ; \mathbb{Z}\right) \cong \mathbb{Z}$.

For a pair of spaces $(X, A)$, we will denote by $H_{i}(X, A)$ and $H^{i}(X, A)$ the $i$-th homology and cohomology groups with rational coefficients, respectively. Using 
the canonical identifications $H_{i}(X, A)=H_{i}(X, A ; \mathbb{Z}) \otimes_{\mathbb{Z}} \mathbb{Q}$ and $H^{i}(X, A)=$ $\operatorname{Hom}_{\mathbb{Z}}\left(H_{i}(X, A ; \mathbb{Z}), \mathbb{Q}\right)$, we will also write

$$
\operatorname{Hur}_{n *}=\operatorname{Hur}_{n} \otimes_{\mathbb{Z}} \mathbb{Q}: \pi_{n}\left(X, A, x_{0}\right) \otimes_{\mathbb{Z}} \mathbb{Q} \rightarrow H_{n}(X, A)
$$

and

$$
\operatorname{Hur}_{n}^{*}=\operatorname{Hom}_{\mathbb{Z}}\left(\operatorname{Hur}_{n}, \mathbb{Q}\right): H^{n}(X, A) \rightarrow \operatorname{Hom}_{\mathbb{Z}}\left(\pi_{n}\left(X, A, x_{0}\right), \mathbb{Q}\right) .
$$

Given maps $X \stackrel{f}{\leftarrow} A \stackrel{g}{\rightarrow} Y$ between topological spaces, we define the homotopy pushout of $f$ and $g$ (see Section 2 in [7]) to be the topological space $X \cup_{A} Y$ defined as the quotient of the disjoint union $A \times[0,1] \sqcup X \sqcup Y$ by the smallest equivalence relation generated by $\{(a, 0) \sim f(a) \mid a \in A\} \cup\{(a, 1) \sim g(a) \mid a \in A\}$. In particular, if $X=\mathrm{pt}$ is the one-point space, then pt $\cup_{A} Y=\operatorname{cone}(g: A \rightarrow Y)=$ cone $(g)$ is just the mapping cone (the homotopy cofiber) of $A \stackrel{g}{\rightarrow} Y$, and throughout the paper we take the cone point pt $\in \operatorname{cone}(g)$ as the canonical basepoint. If, in addition, $Y=A$ and $g=\operatorname{id}_{A}$, then pt $\cup_{A} A=\operatorname{cone}\left(\operatorname{id}_{A}\right)=\operatorname{cone}(A)$ is just the cone of the space $A$. The inclusion $A \times\{1\} \subset A \times[0,1]$ induces a canonical inclusion $A \subset$ cone $(A)$. Moreover, given a map $g: A \rightarrow Y$, the homotopy pushout of $A \stackrel{\operatorname{id}_{A}}{\longleftarrow} A \stackrel{g}{\rightarrow} Y$ is just the mapping cylinder cyl $(g)$ of $g$, and the inclusion $A \times\{0\} \subset A \times[0,1]$ induces a canonical inclusion $A \subset \operatorname{cyl}(g)$.

Let $[M] \in H_{n}(M, \mathbb{Z})$ denote the fundamental class of a closed oriented $n$ manifold $M^{n}$. The image of $[M] \otimes 1$ under the canonical identification $H_{n}(M, \mathbb{Z}) \otimes$ $\mathbb{Q} \cong H_{n}(M)$ will be denoted by $[M]$ as well.

\section{Truncation cones}

Before stating Theorem 4.2, the main result of this section (see Sect. 4.3 for the proof), we explain the necessary notation taken from [7]. Throughout this section, let $\pi: E \rightarrow B$ be a (locally trivial) fiber bundle of closed manifolds with closed manifold fiber $L$ and structure group $G$ such that $B, E$ and $L$ are compatibly oriented. In our applications in Sect. $6, \pi$ will arise as a link bundle of a depth one pseudomanifold $X$, where we utilize the setting of Thom-Mather stratified pseudomanifolds that is considered in the work of Banagl and Chriestenson (see Section 8 in [7]).

Recall that a perversity is a function $\bar{p}:\{2,3, \ldots\} \rightarrow\{0,1, \ldots\}$ which satisfies the Goresky-MacPherson growth conditions $\bar{p}(2)=0$ and $\bar{p}(s) \leq \bar{p}(s+1) \leq$ $\bar{p}(s)+1$ for all $s \in\{2,3, \ldots\}$. We fix two perversities $\bar{p}$ and $\bar{q}$, and require them to be complementary in the sense that $\bar{p}(s)+\bar{q}(s)=s-2=\bar{t}(s)$ for all $s \in\{2,3, \ldots\}$, where $\bar{t}$ is called the top perversity. Let $n-1=\operatorname{dim} E$ and $c=\operatorname{dim} L$ denote the dimensions of the total space and the fiber, respectively. We define the cut-off degrees $k=c-\bar{p}(c+1)$ and $l=c-\bar{q}(c+1)$, and note that $k+l=2 c-\bar{t}(c+1)=c+1$.

Recall from Definition 3.2 in [7] that a $G$-equivariant Moore approximation to $L$ of degree $r$ is a $G$-space $L_{<r}$ together with a $G$-equivariant map $L_{<r} \rightarrow L$ that induces isomorphisms $H_{i}\left(L_{<r}\right) \cong H_{i}(L)$ in degrees $i<r$, 
and such that $H_{i}\left(L_{<r}\right)=0$ in degrees $i \geq r$. We assume that $H_{i}(L)=0$ for $i=\min \{k, l\}, \ldots, \max \{k, l\}-1$, and that the fiber $L$ possesses a $G$-equivariant Moore approximation of degree $k$. Equivalently, there is a map $f_{<}: L_{<} \rightarrow L$ which is a $G$-equivariant Moore approximation to $L$ both of degree $k$ and of degree $l$. This situation is of interest in the important case that $\pi$ is the link bundle of a two strata Witt space, and $\bar{p}=\bar{m}$ and $\bar{q}=\bar{n}$ are the lower middle and upper middle perversities defined by $\bar{m}(s)=\lfloor s / 2\rfloor-1$ and $\bar{n}(s)=\lceil s / 2\rceil-1$ for all $s \in\{2,3, \ldots\}$, respectively (see Section 10 of [7]). In this case it follows that $k=l=(c+1) / 2$ for $c$ odd, and $\min \{k, l\}=c / 2$ and $\max \{k, l\}=c / 2+1$ for $c$ even.

Fix a $G$-equivariant Moore approximation $f_{<}: L_{<} \rightarrow L$ which is both of degree $k$ and of degree $l$. For later reference, we observe that

$$
\widetilde{H}_{i}\left(\operatorname{cone}\left(f_{<}\right)\right)=0, \quad i<\max \{k, l\},
$$

which follows from the long exact sequence on reduced homology induced by the pair $\left(\operatorname{cyl}\left(f_{<}\right), L_{<}\right)$by using the properties of the Moore approximation.

Following the discussion leading to Definition 6.1 in [7], we can consider the induced fiberwise truncation (both of degree $k$ and of degree $l$ )

$$
F_{<}: \mathrm{ft}_{<} E \rightarrow E
$$

Recall that $\mathrm{ft}_{<} E$ is the total space of the fiber bundle $\pi_{<}: \mathrm{ft}_{<} E \rightarrow B$ obtained by replacing the fiber $L$ of $\pi$ with the fiber $L_{<}$(by means of the $G$-action), and $F_{<}: \mathrm{ft}_{<} E \rightarrow E$ is induced by $f_{<}: L_{<} \rightarrow L$ (using $G$-equivariance) in such a way that $\pi \circ F_{<}=\pi_{<}$. The mapping cone of the fiberwise truncation $F_{<}$plays a central role in the theory. In fact, according to Definition 9.1 in [7] the perversity $\bar{p}$ and perversity $\bar{q}$ intersection spaces of a two strata pseudomanifold $X^{n}$ are given by

$$
I X=I^{\bar{p}} X=I^{\bar{q}} X=\operatorname{cone}\left(F_{<}\right) \cup_{E} M,
$$

where $M$ is the $n$-dimensional manifold with boundary $\partial M=E$ that arises as the complement of a suitable tubular neighborhood of $B$ in $X$. (Note that $I X$ is actually defined as the mapping cone of the composition of $F_{<}$with the inclusion $E \subset M$, but this space can be seen to be homeomorphic to cone $\left(F_{<}\right) \cup_{E} M$.) Furthermore, we can characterize the vanishing of the local duality obstructions $\mathcal{O}_{*}(\pi, k, l)$ introduced in Definition 6.8 in [7] in terms of the truncation cone cone $\left(F_{<}\right)$as follows.

Lemma 4.1. We have $\mathcal{O}_{i}(\pi, k, l)=0$ for all $i \notin\{1, \ldots, n-2\}$. Moreover, if $B$ admits a good cover, then we have $\mathcal{O}_{i}(\pi, k, l)=0$ in degree $i \in \mathbb{Z}$ if and only if $x \cup y=0$ in $\widetilde{H}^{n-1}\left(\operatorname{cone}\left(F_{<}\right)\right)$for all $x \in \widetilde{H}^{i}\left(\operatorname{cone}\left(F_{<}\right)\right), y \in \widetilde{H}^{n-1-i}\left(\operatorname{cone}\left(F_{<}\right)\right)$.

Proof. With the fiberwise truncation $F_{<}$being both of degree $k$ and of degree $l$, the local duality obstructions $\mathcal{O}_{*}(\pi, k, l)$ are defined in terms of a certain map $C_{\geq}: E \rightarrow Q_{\geq} E$ constructed from $F_{<}$in Definition 6.3 of [7]. It follows from Definition 6.8 in [7] that we have $\mathcal{O}_{i}(\pi, k, l)=0$ if and only if the cup products $C_{\geq}^{*}\left(x^{\prime}\right) \cup C_{\geq}^{*}\left(y^{\prime}\right)=C_{\geq}^{*}\left(x^{\prime} \cup y^{\prime}\right) \in H^{n-1}(E)$ vanish for all $x^{\prime} \in \widetilde{H}^{i}\left(Q_{\geq} E\right)$ and $y^{\prime} \in \widetilde{H}^{n-1-i}\left(Q_{\geq} E\right)$. To show the claims, we use properties (a) and (b) of $Q_{\geq} E$ 
stated at the beginning of the proof of Theorem 4.2 (see Sect. 4.3 below), from where we also recall that property (a) is only available under the assumption that $B$ admits a good cover. The first claim follows because $Q_{\geq} E$ is path connected. (In fact, we have $Q_{\geq} E \simeq \operatorname{cone}\left(F_{<}\right)$by property (b) of $Q_{\geq} E$, and the truncation cone cone $\left(F_{<}\right)$is path connected by Lemma 4.17 below.) To show the second claim, we first note that the map $C_{\geq}^{*}: H^{n-1}\left(Q_{\geq} E\right) \rightarrow H^{n-1}(E)$ is injective by property (a) of $Q_{\geq} E$ because $B$ admits a good open cover by assumption. Then, the second claim follows from the fact that the cohomology rings $H^{*}\left(Q_{\geq} E\right)$ and $H^{*}\left(\operatorname{cone}\left(F_{<}\right)\right)$are isomorphic by property (b) of $Q_{\geq} E$.

We state the main result of this section (see Sect. 4.3 for the proof).

Theorem 4.2. Let $n \geq 3$ be an integer. Let $\pi: E^{n-1} \rightarrow B$ be a fiber bundle of closed manifolds with closed manifold fiber $L$ and structure group $G$ such that $B, E$ and $L$ are compatibly oriented. Suppose that $B$ admits a good open cover (this holds whenever $B$ is smooth or at least $P L)$. Let $\bar{p}$ and $\bar{q}$ be complementary perversities, and set $k=c-\bar{p}(c+1)$ and $l=c-\bar{q}(c+1)$, where $c=\operatorname{dim}$ L. Suppose that the fiber $L$ possesses a G-equivariant Moore approximation $f_{<}: L_{<} \rightarrow L$ which is both of degree $k$ and of degree $l$. Let $F_{<}: \mathrm{ft}_{<} E \rightarrow E$ denote the induced fiberwise truncation of $\pi$. Then, the following statements are equivalent:

(i) There exists an attaching map

$$
\phi:\left(S^{n-1}, s_{0}\right) \rightarrow\left(\operatorname{cone}\left(F_{<}\right), \mathrm{pt}\right)
$$

and a lift $\left[e_{\phi}\right] \in H_{n}$ (cone $\left.\left(F_{<}\right) \cup_{\phi} D^{n}, E\right)$ of the fundamental class $[E] \in$ $H_{n-1}(E)$ under the connecting homomorphism

$$
\partial_{n}: H_{n}\left(\operatorname{cone}\left(F_{<}\right) \cup_{\phi} D^{n}, E\right) \rightarrow H_{n-1}(E)
$$

such that (cone $\left.\left(F_{<}\right) \cup_{\phi} D^{n}, E\right)$ is a rational Poincaré duality pair of dimension $n$ with orientation class $\left[e_{\phi}\right]$ (see Definition 4.7$)$.

(ii) The image of the fundamental class $[E] \in H_{n-1}(E)$ under the map

$$
H_{n-1}(E) \rightarrow H_{n-1}\left(\operatorname{cone}\left(F_{<}\right)\right)
$$

induced by the inclusion $E \subset \operatorname{cone}\left(F_{<}\right)$is contained in the image of the rational Hurewicz homomorphism

$$
\operatorname{Hur}_{n-1 *}: \pi_{n-1}\left(\operatorname{cone}\left(F_{<}\right), \mathrm{pt}\right) \otimes_{\mathbb{Z}} \mathbb{Q} \rightarrow H_{n-1}\left(\operatorname{cone}\left(F_{<}\right)\right) .
$$

Furthermore, if either of the above statements holds, then $\mathcal{O}_{i}(\pi, k, l)=0$ for all $i$.

Remark 4.3. (choice of basepoints) We point out that the proof of the equivalence of the statements $(i)$ and $(i i)$ in Theorem 4.2 (see Sect. 4.3) works out for any choice of basepoint $x_{0} \in \operatorname{cone}\left(F_{<}\right)$, not just for the cone point $x_{0}=$ pt used in Theorem 4.2. Moreover, if statement $(i i)$ is valid for a specific choice of basepoint, then it is valid for any choice of basepoint of $\operatorname{cone}\left(F_{<}\right)$. (In fact, $\operatorname{cone}\left(F_{<}\right)$is path connected by Lemma 4.17, and a change-of-basepoint transformation on homotopy groups covers the identity map on homology groups under the Hurewicz homomorphism.) 
Remark 4.4. (CW structures) In applications it might be necessary to know that the Poincaré duality pair provided by statement (i) in Theorem 4.2 is a CW pair. To achieve this, we assume that the spaces $B, L$, and $L_{<}$carry $\mathrm{CW}$ structures, and that the map $f_{<}: L_{<} \rightarrow L$ is cellular. Then, note that $E$ and $\mathrm{ft}_{<} E$ inherit natural CW structures in such a way that the induced fiberwise truncation $F_{<}: \mathrm{ft}_{<} E \rightarrow E$ is cellular. Thus, the truncation cone $\operatorname{cone}\left(F_{<}\right)$has a cell structure. Finally, by applying the cellular approximation theorem to the map $\phi$ in Lemma 4.5, we may without loss of generality assume that the map $\phi$ in statement (i) of Theorem 4.2 is cellular. Hence, it follows that $\left(\operatorname{cone}\left(F_{<}\right) \cup_{\phi} D^{n}, E\right)$ is a CW pair.

\subsection{The rational Hurewicz homomorphism}

The following lemma is a slight modification of Lemma 3.8 in [23, p. 248], and will be used in the proofs of Theorem 4.2 (see Sect. 4.3) and Theorem 6.3.

Lemma 4.5. Let $n \geq 3$ be an integer. Let $\phi:\left(S^{n-1}, s_{0}\right) \rightarrow\left(X, x_{0}\right)$ be a map of pointed spaces, and let $X^{\phi}=\mathrm{cone}(\phi)$ denote the associated mapping cone. Then for any class $x \in H_{n-1}(X)$ the following statements are equivalent:

(i) There exists $q \in \mathbb{Q}$ such that the element $[\phi] \otimes q \in \pi_{n-1}\left(X, x_{0}\right) \otimes_{\mathbb{Z}} \mathbb{Q}$ is mapped to the class $x \in H_{n-1}(X)$ under the Hurewicz homomorphism

$$
\operatorname{Hur}_{n-1 *}: \pi_{n-1}\left(X, x_{0}\right) \otimes_{\mathbb{Z}} \mathbb{Q} \rightarrow H_{n-1}(X) .
$$

(ii) The class $x \in H_{n-1}(X)$ lies in the image of the connecting homomorphism

$$
\partial_{n}: H_{n}\left(X^{\phi}, X\right) \rightarrow H_{n-1}(X) .
$$

Proof. Consider the commutative diagram

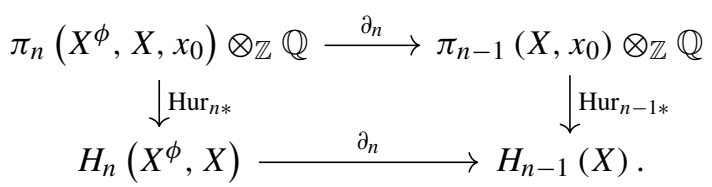

By construction of $X^{\phi}$ we have a map of pointed pairs

$$
(\Phi, \phi):\left(D^{n}, S^{n-1}, s_{0}\right) \rightarrow\left(X^{\phi}, X, x_{0}\right) .
$$

Consider the induced element $[\Phi] \otimes 1 \in \pi_{n}\left(X^{\phi}, X, x_{0}\right) \otimes_{\mathbb{Z}} \mathbb{Q}$. The homomorphism

$$
\partial_{n}: \pi_{n}\left(X^{\phi}, X, x_{0}\right) \otimes_{\mathbb{Z}} \mathbb{Q} \rightarrow \pi_{n-1}\left(X, x_{0}\right) \otimes_{\mathbb{Z}} \mathbb{Q}
$$

maps $[\Phi] \otimes 1$ to the element $[\phi] \otimes 1 \in \pi_{n-1}\left(X, x_{0}\right) \otimes_{\mathbb{Z}} \mathbb{Q}$. The homomorphism

$$
\operatorname{Hur}_{n *}: \pi_{n}\left(X^{\phi}, X, x_{0}\right) \otimes_{\mathbb{Z}} \mathbb{Q} \rightarrow H_{n}\left(X^{\phi}, X\right)
$$


maps $[\Phi] \otimes 1$ to a generator of $H_{n}\left(X^{\phi}, X\right) \cong \tilde{H}_{n}\left(X^{\phi} / X\right) \cong \tilde{H}_{n}\left(S^{n}\right) \cong \mathbb{Q}$. (Here, the isomorphism $H_{n}\left(X^{\phi}, X\right) \cong \widetilde{H}_{n}\left(X^{\phi} / X\right)$ holds by Proposition 2.22 in [22, p. 124].) Thus, if statement $(i)$ holds, then we have

$$
x=\operatorname{Hur}_{n-1 *}([\phi] \otimes q)=\operatorname{Hur}_{n-1 *}\left(\partial_{n}([\Phi] \otimes q)\right)=\partial_{n}\left(\operatorname{Hur}_{n *}([\Phi] \otimes q)\right),
$$

and statement $(i i)$ follows. Conversely, if statement ( $i i)$ holds, then there exists $q \in \mathbb{Q}$ such that

$$
x=\partial_{n}\left(q \cdot \operatorname{Hur}_{n *}([\Phi] \otimes 1)\right)=\operatorname{Hur}_{n-1 *}\left(\partial_{n}([\Phi] \otimes q)\right)=\operatorname{Hur}_{n-1 *}([\phi] \otimes q),
$$

and statement $(i)$ follows.

Remark 4.6. For $n=2$, Lemma 4.5 and its proof remain valid after replacing $\pi_{1}\left(X, x_{0}\right)$ by its abelianization $\pi_{1}\left(X, x_{0}\right)_{a b}$. However, the integer $n$ will arise in our applications in Sect. 6 as the dimension of depth one pseudomanifolds. As their singular strata are required to have codimension at least 2 (see Sect. 6.1) and the case of point strata is covered by [23], we will generally assume that $n \geq 3$ throughout the paper.

\subsection{Rational Poincaré duality pairs}

We recall the fundamental concept of Poincaré duality pairs of spaces (compare Section 3.1 in [23] and Section I.2 in [11]). In this paper we do not require cell structures on our spaces, but see Remark 4.4.

Definition 4.7. A pair $(A, B)$ is called (rational) Poincaré duality pair of dimension $n$ if

(i) all homology groups $H_{r}(A)$ and $H_{r}(B), r \in \mathbb{Z}$, are of finite rank, and

(ii) there exists a class $a \in H_{n}(A, B)$ such that

$$
-\cap a: H^{r}(A) \rightarrow H_{n-r}(A, B)
$$

is an isomorphism for all $r \in \mathbb{Z}$. Any such class $a \in H_{n}(A, B)$ will be called an orientation class for $(A, B)$.

Remark 4.8. It is well-known (see Remark 3.2 in [23, p. 245] and Corollary I.2.3 in $[11$, p. 8]) that for a Poincaré duality pair $(A, B)$ of dimension $n$ which is equipped with an orientation class $a \in H_{n}(A, B)$, the associated oriented boundary $B=(B, \emptyset)$ is a Poincaré duality pair of dimension $n-1$ by means of the orientation class $\partial_{n} a \in H_{n-1}(B)$.

In the following, a Poincaré duality pair of the form $A=(A, \emptyset)$ will be called a Poincaré space.

We state our main technical result, which is a careful extension of Lemma 3.7 in [23, p. 247]. Our purpose is to cover also the case of depth one pseudomanifolds having non-isolated singular strata as considered in [7]. 
Proposition 4.9. Let $Z$ be a Poincaré space of dimension $n-1>0$ with orientation class $[Z] \in H_{n-1}(Z)$. Let $f: Y \rightarrow Z$ be a map from a space $Y$ to $Z$ such that the induced map $f_{*}: H_{0}(Y) \rightarrow H_{0}(Z)$ is bijective, and let $X=\operatorname{cone}(f)$ denote the mapping cone of $f$. Suppose that for every $r \in \mathbb{Z}$ the following conditions hold:

(1) The inclusion $Z \subset X$ induces a surjective homomorphism $H_{r}(Z) \rightarrow H_{r}(X)$.

(2) The rational vector spaces $H_{r}(Y)$ and $\widetilde{H}_{n-r-1}(X)$ have the same rank.

Then, for any space $X^{\phi}=\operatorname{cone}(\phi)$ given by the mapping cone of some map $\phi: S^{n-1} \rightarrow X$ the following statements are equivalent:

(i) There exists a lift $\left[e_{\phi}\right] \in H_{n}\left(X^{\phi}, Z\right)$ of the orientation class $[Z] \in H_{n-1}(Z)$ under the connecting homomorphism $\partial_{n}: H_{n}\left(X^{\phi}, Z\right) \rightarrow H_{n-1}(Z)$ such that the pair $\left(X^{\phi}, Z\right)$ is a Poincaré duality pair of dimension $n$ with orientation class $\left[e_{\phi}\right]$.

(ii) The orientation class $[Z] \in H_{n-1}(Z)$ of $Z$ lies in the image of the connecting homomorphism $\partial_{n}: H_{n}\left(X^{\phi}, Z\right) \rightarrow H_{n-1}(Z)$.

(iii) The image of the orientation class $[Z] \in H_{n-1}(Z)$ of $Z$ under the map $H_{n-1}(Z) \rightarrow H_{n-1}(X)$ induced by the inclusion $Z \subset X$ lies in the image of the connecting homomorphism $\partial_{n}: H_{n}\left(X^{\phi}, X\right) \rightarrow H_{n-1}(X)$.

Proof. (ii) $\Leftrightarrow$ (iii). The inclusion of pairs $\left(X^{\phi}, Z\right) \subset\left(X^{\phi}, X\right)$ induces the commutative diagram

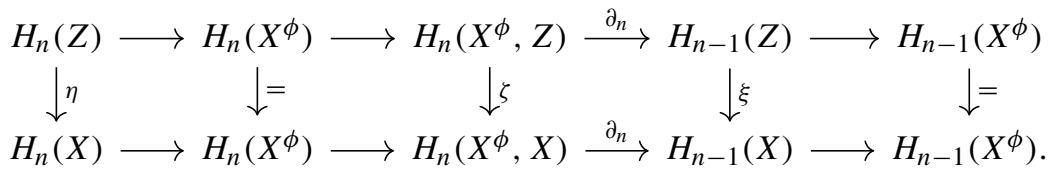

Let us show that $\eta$ and $\xi$ are isomorphisms. First, note that $\eta$ and $\xi$ are surjective homomorphisms of finite dimensional vector spaces by assumption (1) applied for $r=n$ and $r=n-1$, respectively, as well as by Definition 4.7(i). Next, observe that $H_{n}(Z) \cong H_{-1}(Z)=0$ and $H_{n-1}(Z) \cong H_{0}(Z)$ because $Z$ is a Poincaré space of dimension $n-1$. Moreover, using that $H_{0}(Y) \cong H_{0}(Z)$ and that $n-1>0$, we obtain $H_{n-1}(X)=\widetilde{H}_{n-1}(X) \cong H_{0}(Y) \cong H_{0}(Z)$ by assumption (2) applied for $r=0$. All in all, we have shown that $\eta$ and $\xi$ are isomorphisms. Finally, the five lemma implies that the map $\zeta$ in the above diagram is an isomorphism as well, and the equivalence $($ ii $) \Leftrightarrow$ (iii) follows.

Remark 4.10. For future reference we note that assuming either (ii) or (iii) in Proposition 4.9, we can show that the connecting homomorphisms in the diagram

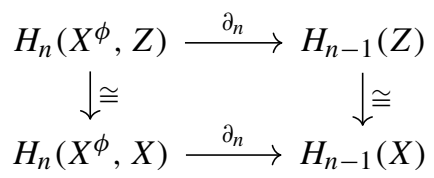

are both injective because $H_{n}\left(X^{\phi}, Z\right) \cong H_{n}\left(X^{\phi}, X\right) \cong \mathbb{Q}$, and they are non-trivial. (Indeed, observe that we have

$$
H_{n}\left(X^{\phi}, X\right) \cong \widetilde{H}_{n}\left(X^{\phi} / X\right) \cong \widetilde{H}_{n}\left(S^{n}\right) \cong \mathbb{Q} .
$$


Here, the isomorphism $H_{n}\left(X^{\phi}, X\right) \cong \widetilde{H}_{n}\left(X^{\phi} / X\right)$ holds by Proposition 2.22 in [22, p. 124]. Note that for any map $\alpha: C \rightarrow D$, the pair (cone $(\alpha), D)$ is a good pair, that is, $D$ is a nonempty closed subset of $\operatorname{cone}(\alpha)$ that is a deformation retract of some neighborhood in cone $(\alpha)$.)

$(i) \Rightarrow(i i)$. This implication is clear because statement $(i)$ implies that $[Z]=$ $\partial_{n}\left(\left[e_{\phi}\right]\right)$.

(ii) $\Rightarrow(i)$. By statement (ii) there exists an element $\left[e_{\phi}\right] \in H_{n}\left(X^{\phi}, Z\right)$ such that $\partial_{n}\left(\left[e_{\phi}\right]\right)=[Z] \in H_{n-1}(Z)$. (In fact, $e_{\phi}$ is uniquely determined because $\partial_{n}$ is injective by Remark 4.10.) We claim that the homomorphism

$$
-\cap\left[e_{\phi}\right]: H^{r}\left(X^{\phi}\right) \rightarrow H_{n-r}\left(X^{\phi}, Z\right)
$$

is an isomorphism for every $r \in \mathbb{Z}$. (It is clear by assumption (1) that all rational homology groups of $X^{\phi}$ have finite rank, so that $\left(X^{\phi}, Z\right)$ will then be a Poincaré duality pair of dimension $n$ according to Definition 4.7.) In general, observe that every element $\alpha \in H_{n}\left(X^{\phi}, Z\right)$ gives by Proposition I.1.4(ii) in [11, p. 4] rise to a commutative diagram

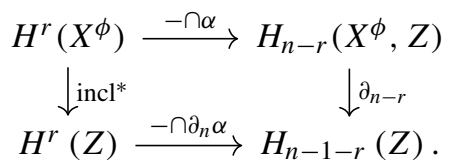

If we specialize to $\alpha=\left[e_{\phi}\right]$, then the lower horizontal homomorphism $-\cap[Z]$ is an isomorphism because $Z$ is a Poincaré space of dimension $n-1$. Thus, in order to show that the upper horizontal row of the above diagram is an isomorphism, it suffices to verify that for every $r \in \mathbb{Z}$, the following two assertions (a) and (b) hold. (In fact, by assertion (a) below, the map incl*: $H^{r}\left(X^{\phi}\right) \rightarrow H^{r}(Z)$ in the above diagram is injective. Since the lower horizontal map $-\cap[Z]$ is an isomorphism, it follows that the upper horizontal map $-\cap\left[e_{\phi}\right]$ is injective as well, and thus an isomorphism in view of assertion (b) below.)

(a) The inclusion $Z \subset X^{\phi}$ induces a surjective map $H_{r}(Z) \rightarrow H_{r}\left(X^{\phi}\right)$. In view of assumption (1), our claim (a) is in fact equivalent to showing that the inclusion $X \subset X^{\phi}$ induces a surjective homomorphism $H_{r}(X) \rightarrow H_{r}\left(X^{\phi}\right)$. We compute

$$
H_{r}\left(X^{\phi}, X\right) \cong \widetilde{H}_{r}\left(X^{\phi} / X\right) \cong \widetilde{H}_{r}\left(S^{n}\right) \cong \begin{cases}\mathbb{Q}, & r=n, \\ 0, & r \neq n .\end{cases}
$$

Thus, for $r \neq n$, the claim follows from the exactness of

$$
H_{r}(X) \rightarrow H_{r}\left(X^{\phi}\right) \rightarrow H_{r}\left(X^{\phi}, X\right) .
$$

For $r=n$ we consider the exact sequence

$$
H_{n}(X) \rightarrow H_{n}\left(X^{\phi}\right) \rightarrow H_{n}\left(X^{\phi}, X\right) \stackrel{\partial_{n}}{\rightarrow} H_{n-1}(X) .
$$

Since the connecting homomorphism $\partial_{n}$ is injective by Remark 4.10, we conclude that the homomorphism $H_{n}(X) \rightarrow H_{n}\left(X^{\phi}\right)$ is surjective. 
Remark 4.11. Suppose that either (ii) or (iii) holds in Proposition 4.9. Then, for future reference, we note that an inspection of the homology long exact sequence of the pair $\left(X^{\phi}, X\right)$ combined with the above information implies that the inclusion $X \subset X^{\phi}$ induces an isomorphism $H_{r}(X) \stackrel{\cong}{\longrightarrow} H_{r}\left(X^{\phi}\right)$ for $r \neq n-1$, whereas the induced map $H_{n-1}(X) \rightarrow H_{n-1}\left(X^{\phi}\right)$ is surjective with kernel isomorphic to $H_{n}\left(X^{\phi}, X\right) \cong \mathbb{Q}$.

(b) The rational vector spaces $H_{r}\left(X^{\phi}\right)$ and $H_{n-r}\left(X^{\phi}, Z\right)$ have the same (finite) rank.

To prove assertion (b), it suffices to show that

$$
H_{r}\left(X^{\phi}, Z\right) \cong \begin{cases}\widetilde{H}_{r-1}(Y), & r \neq n, \\ H_{n-1}(Y) \oplus \mathbb{Q}, & r=n .\end{cases}
$$

(In fact, writing $\delta_{i, i}=1$ and $\delta_{i, j}=0$ for $i \neq j$, we then obtain

$$
\begin{aligned}
\operatorname{rank} H_{n-r}\left(X^{\phi}, Z\right) & \stackrel{n \neq 1}{=} \operatorname{rank} H_{n-r-1}(Y)-\delta_{n-r, 1}+\delta_{n-r, n} \\
& \stackrel{(2)}{=} \operatorname{rank} \widetilde{H}_{r}(X)-\delta_{r, n-1}+\delta_{r, 0} \\
& =\operatorname{rank} H_{r}(X)-\delta_{r, n-1} \\
& \stackrel{4.11}{=} \operatorname{rank} H_{r}\left(X^{\phi}\right)
\end{aligned}
$$

where we have used $n \neq 1$, as well as assumption (2) and Remark 4.11.)

The inclusion of pairs $(X, Z) \subset\left(X^{\phi}, Z\right)$ induces the commutative diagram

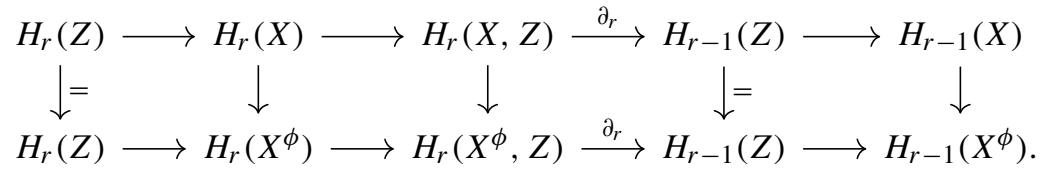

Observe that for all $r \in \mathbb{Z}$,

$$
H_{r}(X, Z) \cong \widetilde{H}_{r}(X / Z) \cong \widetilde{H}_{r}(\Sigma Y) \cong \widetilde{H}_{r-1}(Y) .
$$

(Here, the isomorphism $H_{r}(X, Z) \cong \widetilde{H}_{r}(X / Z)$ holds by Proposition 2.22 in [22, p. 124] because $X$ is the cone of $f: Y \rightarrow Z$.) Since by Remark 4.11 the inclusion $X \subset X^{\phi}$ induces an isomorphism $H_{r}(X) \stackrel{\cong}{\longrightarrow} H_{r}\left(X^{\phi}\right)$ for $r \neq n-1$, the claim follows for $r \notin\{n-1, n\}$ from the five lemma applied to the above diagram. We check the remaining cases:

- In the case $r=n-1$, we note that in the above diagram the homomorphism $H_{n-1}(Z) \rightarrow H_{n-1}(X)$ is surjective by assumption (1), and the homomorphism $H_{n-1}(X) \rightarrow H_{n-1}\left(X^{\phi}\right)$ is surjective by Remark 4.11 . Thus, we obtain the following simplified commutative diagram with exact rows:

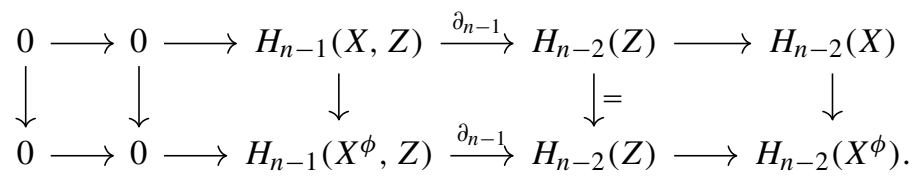


Finally, as the right vertical arrow $H_{n-2}(X) \rightarrow H_{n-2}\left(X^{\phi}\right)$ is an isomorphism by Remark 4.11, the five lemma yields

$$
H_{n-1}\left(X^{\phi}, Z\right) \cong H_{n-1}(X, Z) \cong \widetilde{H}_{n-2}(Y) .
$$

- In the case $r=n$, we consider the following portion of the homology exact sequence of the pair $(X, Z)$ :

$$
H_{n}(X) \rightarrow H_{n}(X, Z) \rightarrow H_{n-1}(Z) \rightarrow H_{n-1}(X)
$$

Note that $H_{n-1}(X) \cong H_{0}(Y)$ and $H_{n}(X) \cong 0$ by assumption (2). As the homomorphism $H_{n-1}(Z) \rightarrow H_{n-1}(X)$ is surjective by assumption (1), we obtain

$$
H_{n-1}(Z) \cong H_{n}(X, Z) \oplus H_{n-1}(X) \cong H_{n-1}(Y) \oplus H_{0}(Y) .
$$

Hence, it follows from $H_{n-1}(Z) \cong H_{0}(Z) \cong H_{0}(Y)$ that $H_{n-1}(Y)=0$. On the other hand, $H_{n}\left(X^{\phi}, Z\right) \cong \mathbb{Q}$ according to Remark 4.10.

Remark 4.12. Suppose that either (ii) or (iii) holds in Proposition 4.9. Then, for future reference, we note that the inclusion of pairs $(X, Z) \subset\left(X^{\phi}, Z\right)$ induces an isomorphism $H_{r}(X, Z) \stackrel{\cong}{\longrightarrow} H_{r}\left(X^{\phi}, Z\right)$ for $r \neq n$, whereas $H_{n}\left(X^{\phi}, Z\right) \cong$ $H_{n}(X, Z) \oplus \mathbb{Q}$.

By invoking Lemma 4.5, we obtain the following

Corollary 4.13. If $f: Y \rightarrow Z$ and $\left(X, x_{0}\right)=($ cone $(f)$, pt) satisfy all assumptions of Proposition 4.9, then the following statements are equivalent:

(i) There exists a map $\phi:\left(S^{n-1}, s_{0}\right) \rightarrow\left(X, x_{0}\right)$ such that statement (i) of Proposition 4.9 holds for $X^{\phi}=\operatorname{cone}(\phi)$.

(ii) The image of the orientation class $[Z] \in H_{n-1}(Z)$ of $Z$ under the map $H_{n-1}(Z) \rightarrow H_{n-1}(X)$ induced by the inclusion $Z \subset X$ lies in the image of the rational Hurewicz homomorphism

$$
\operatorname{Hur}_{n-1 *}: \pi_{n-1}\left(X, x_{0}\right) \otimes_{\mathbb{Z}} \mathbb{Q} \rightarrow H_{n-1}(X) .
$$

Proof. By combining the equivalence (i) $\Leftrightarrow$ (iii) of Proposition 4.9 and the equivalence (ii) $\Leftrightarrow$ (i) of Lemma 4.5 , we conclude that statement (i) is equivalent to the following statement. There exist a map $\phi:\left(S^{n-1}, s_{0}\right) \rightarrow\left(X, x_{0}\right)$ and $q \in \mathbb{Q}$ such that $\operatorname{Hur}_{n-1 *}([\phi] \otimes q) \in H_{n-1}(X)$ equals the image of the orientation class $[Z] \in H_{n-1}(Z)$ of $Z$ under the map $H_{n-1}(Z) \rightarrow H_{n-1}(X)$ induced by the inclusion $Z \subset X$. But since every element of $\pi_{n-1}\left(X, x_{0}\right) \otimes_{\mathbb{Z}} \mathbb{Q}$ can be written in the form $[\phi] \otimes q$ for suitable $\phi$ and $q$, the previous statement is equivalent to statement (ii), and the claim follows. 


\subsection{Proof of Theorem 4.2}

Following Definition 6.2 in [7], we define the fiberwise cotruncation $\mathrm{ft}_{\geq} E$ (both of degree $k$ and of degree $l$ ) of the fiber bundle $\pi: E \rightarrow B$ as the homotopy pushout of the diagram

$$
B \stackrel{\pi_{<}}{\longleftarrow} \mathrm{ft}_{<} E \stackrel{F_{<}}{\longrightarrow} E .
$$

In Definition 6.3 of [7] an auxiliary space $Q_{\geq} E$ together with a structure map $C_{\geq}: E \rightarrow Q_{\geq} E$ is introduced. In Section 6 of [7] the following properties of $Q_{\geq} E$ are derived (the assumption that $B$ admits a good cover is needed to deduce item (a) and (c) because they are both based on Proposition 6.5 in [7]):

(a) By Lemma 6.6 in [7], the map $C_{\geq}: E \rightarrow Q_{\geq} E$ induces for every $r \in \mathbb{Z}$ a surjective homomorphism $C_{\geq *}: H_{r}(E) \rightarrow H_{r}\left(Q_{\geq} E\right)$.

(b) By the proof of Lemma 6.6 in [7], there exists a diagram

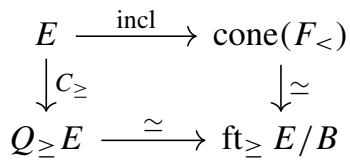

which commutes on the nose. In particular, $Q_{\geq} E$ and cone $\left(F_{<}\right)$are homotopy equivalent spaces.

(b) According to Proposition 6.7 in [7], the rational vector spaces $H_{r}$ ( $\mathrm{ft}_{<} E$ ) and $\widetilde{H}_{n-r-1}\left(Q_{\geq} E\right)$ have for every $r \in \mathbb{Z}$ the same rank.

Let us prove the equivalence $(i) \Leftrightarrow$ (ii) claimed by Theorem 4.2. For this purpose, it suffices to apply Corollary 4.13 to the map $f: Y \rightarrow Z$ given by $F_{<}: \mathrm{ft}_{<} E \rightarrow E$. Note that the space $Z=E$ is a Poincare space with orientation class given by $[Z]=[E] \in H_{n-1}(Z)$ because $E$ is a closed oriented manifold. Moreover, note that the map $F_{<}: \mathrm{ft}_{<} E \rightarrow E$ induces a bijection $F_{<}: H_{0}\left(\mathrm{ft}_{<} E\right) \rightarrow H_{0}(E)$. Taking $\left(X, x_{0}\right)=\left(\operatorname{cone}\left(F_{<}\right), \mathrm{pt}\right)$ to be the mapping cone of the map $f=F_{<}$, we see that condition (1) of Proposition 4.9 holds by properties (a) and (b) of $Q_{\geq} E$, and that condition (2) of Proposition 4.9 holds by properties (b) and (c) of $Q_{\geq} E$. Thus, all assumptions of Corollary 4.13 hold, and the desired equivalence $(i) \Leftrightarrow(i i)$ follows.

We continue to use the notation $f: Y \rightarrow Z$ for the map $F_{<}: \mathrm{ft}_{<} E \rightarrow E$, and write $X=\operatorname{cone}\left(F_{<}\right)$for the mapping cone of $f=F_{<}$. From now on we suppose that either of the equivalent statements of Theorem 4.2 holds. Fix $r \in$ $\{1, \ldots, n-2\}$. We have to show that the local duality obstruction $\mathcal{O}_{n-1-r}(\pi, k, l)$ vanishes (see Lemma 4.1). By statement $(i)$ there is a map $\phi: S^{n-1} \rightarrow X$ and a lift $\left[e_{\phi}\right] \in H_{n}\left(X^{\phi}, Z\right)$ of the orientation class $[Z] \in H_{n-1}(Z)$ such that $\left(X^{\phi}, Z\right)$ is a rational Poincaré duality pair of dimension $n$ with orientation class $\left[e_{\phi}\right]$. By Theorem I.2.2 in $[11$, p. 8], there is the following commutative diagram:

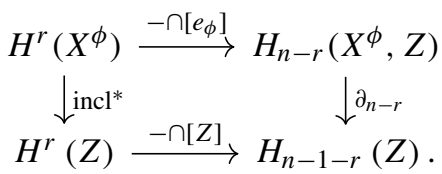


Factoring the inclusion $Z \subset X^{\phi}$ as $Z \subset X \subset X^{\phi}$, and using the inclusion of pairs $(X, Z) \subset\left(X^{\phi}, Z\right)$, we can extend the diagram to a commutative diagram as follows:

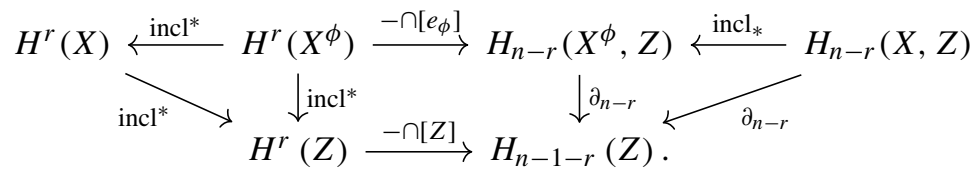

Since for $r \in\{1, \ldots, n-2\}$ the inclusions $X \subset X^{\phi}$ and $(X, Z) \subset\left(X^{\phi}, Z\right)$ induce isomorphisms $H^{r}\left(X^{\phi}\right) \stackrel{\cong}{\longrightarrow} H^{r}(X)$ by Remark 4.11 and $H_{n-r}(X, Z) \stackrel{\cong}{\longrightarrow}$ $H_{n-r}\left(X^{\phi}, Z\right)$ by Remark 4.12, respectively, we obtain a commutative diagram of the form

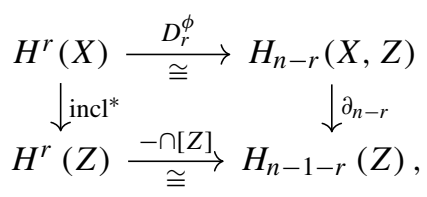

in which $D_{r}^{\phi}$ is the unique isomorphism such that the diagram

$$
\begin{gathered}
H^{r}(X) \stackrel{D_{r}^{\phi}}{\cong} H_{n-r}(X, Z) \\
\cong \uparrow \text { incl* } \\
H^{r}\left(X^{\phi}\right) \stackrel{-\cap\left[e_{\phi}\right]}{\cong} H_{n-r}\left(X^{\phi}, Z\right)
\end{gathered}
$$

commutes. We claim that diagram (3) is part of a commutative diagram of the form

$$
\begin{aligned}
& H^{r}\left(\mathrm{ft}_{\geq} E / B\right) \stackrel{\cong}{\longrightarrow} H^{r}(X) \stackrel{D_{r}^{\phi}}{\cong} H_{n-r}(X, Z) \stackrel{\cong}{\cong} H_{n-r}(\operatorname{cone}(Y), Y)
\end{aligned}
$$

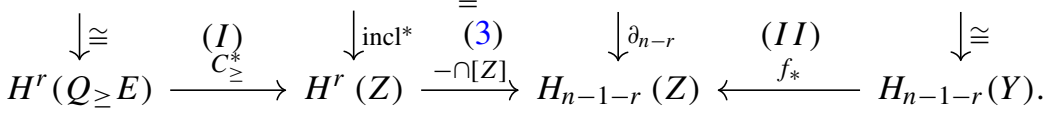

In fact, the commutative square $(I)$ exists by property $(b)$ of $Q_{\geq} E$, and the commutative square $(I I)$ is given by the square $(I I)^{\prime}$ in the following commutative diagram, which is induced by the canonical map of pairs $(\widetilde{f}, f):(\operatorname{cone}(Y), Y) \rightarrow$ $(X, Z)$ :

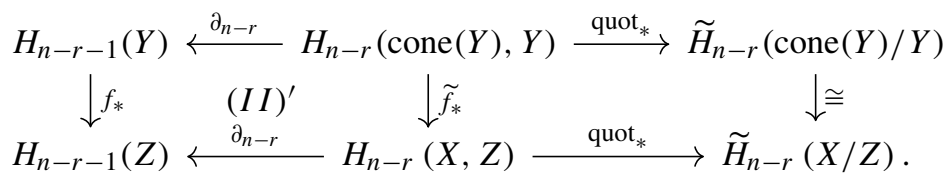

(Note that $\widetilde{f}_{*}$ is an isomorphism because the horizontal arrows induced by the quotient maps $(\operatorname{cone}(Y), Y) \rightarrow(\operatorname{cone}(Y) / Y, \mathrm{pt})$ and $(X, Z) \rightarrow(X / Z, \mathrm{pt})$ are isomorphisms (see Proposition 2.22 in [22, p. 124], and note that (cone $(Y), Y$ ) and $(X, Z)$ are good pairs). Moreover, the connecting homomorphism

$$
\partial_{n-r}: H_{n-r}(\text { cone }(Y), Y) \rightarrow H_{n-r-1}(Y)
$$


is an isomorphism because cone $(Y)$ is contractible, and $r \neq n-1$.)

All in all, we obtain a commutative diagram

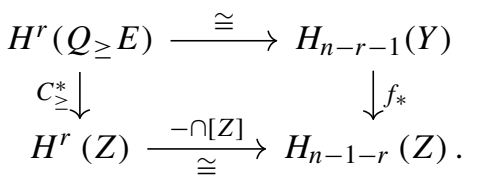

After applying the functor $\operatorname{Hom}_{\mathbb{Q}}(-, \mathbb{Q})$, we conclude from Proposition 6.9 in [7] that $\mathcal{O}_{n-1-r}(\pi, k, l)$ vanishes.

This completes the proof of Theorem 4.2.

Remark 4.14. The isomorphism $D_{r}^{\phi}$ in diagram (4) does actually not depend on $\phi$. In fact, the isomorphism $H^{r}\left(Q_{\geq} E\right) \stackrel{\cong}{\rightrightarrows} H_{n-r-1}(Y)$ in diagram (5) is unique according to the last statement of Proposition 6.9 in [7].

\subsection{Connectivity of truncation cones}

Recall from the beginning of Sect. 4 that $\pi: E \rightarrow B$ is a (locally trivial) fiber bundle of closed manifolds with closed manifold fiber $L$ and structure group $G$ such that $B, E$ and $L$ are compatibly oriented. The dimensions of the total space and the fiber are $n-1=\operatorname{dim} E$ and $c=\operatorname{dim} L$, respectively. Using the given perversities $\bar{p}$ and $\bar{q}$, we have the cut-off degrees $k=c-\bar{p}(c+1)$ and $l=c-\bar{q}(c+1)$. Recall that $f_{<}: L_{<} \rightarrow L$ is a $G$-equivariant Moore approximation which is both of degree $k$ and of degree $l$, and $F_{<}: \mathrm{ft}_{<} E \rightarrow E$ is the associated fiberwise truncation.

In order to be able to apply rational homotopy theory to truncation cones in Sect. 5, we study the connectivity properties of truncation cones in the present section.

The following result shows that the reduced homology of truncation cones vanishes in low degrees.

Lemma 4.15. If $B$ admits a good cover (e.g., if $B$ is smooth or at least $P L)$, then the homology of cone $\left(F_{<}\right)$satisfies $\widetilde{H}_{i}\left(\operatorname{cone}\left(F_{<}\right)\right)=0$ for $i<\max \{k, l\}$.

Proof. We employ the local to global technique based on precosheaves as presented in Section 4 of [7], and assume familiarity with the notation and definitions used therein.

Our argument requires a slight modification of Proposition 4.4 of [7] that applies to finite sequences of $\delta$-compatible morphisms between precosheaves instead of inifinite sequences (see Proposition 4.16 below). For this purpose, consider an open cover $\mathcal{U}$ of the topological space $B$. Let $\tau \mathcal{U}$ denote the category whose objects are unions of finite intersections of open sets in $\mathcal{U}$, and whose morphisms are inclusions. On the product category $\tau \mathcal{U} \times \tau \mathcal{U}$, consider the functors $\cap, \cup: \tau \mathcal{U} \times \tau \mathcal{U} \rightarrow \tau \mathcal{U}$ induced by intersection and union of open sets, respectively. For $i=1,2$, projection to the $i$-th factor determines a projection functor $p_{i}: \tau \mathcal{U} \times \tau \mathcal{U} \rightarrow \tau \mathcal{U}$. We also need for $i=1,2$ the natural transformations $j_{i}: p_{i} \rightarrow \cup$ and $\iota_{i}: \cap \rightarrow p_{i}$ induced by the 
inclusions $U, V \subset U \cup V$ and $U \cap V \subset U, V$, respectively. Now consider a finite sequence $\mathcal{F}_{i}=\mathcal{F}_{0}, \ldots, \mathcal{F}_{N}$ of precosheaves on $B$. Slightly modifying Definition 4.3 in [7], we say that the finite sequence $\mathcal{F}_{i}$ satisfies the $\mathcal{U}$-Mayer-Vietoris property if there are natural transformations of functors on $\tau \mathcal{U} \times \tau \mathcal{U}$,

$$
\delta_{i}^{\mathcal{F}}: \mathcal{F}_{i} \circ \cup \rightarrow \mathcal{F}_{i-1} \circ \cap, \quad 1 \leq i \leq N
$$

such that for every pair of open sets $U, V \in \tau \mathcal{U}$ the following sequence is exact:

$$
\begin{gathered}
\mathcal{F}_{N}(U \cap V) \stackrel{\left(l_{1}^{N}, l_{2}^{N}\right)}{\longrightarrow} \mathcal{F}_{N}(U) \oplus \mathcal{F}_{N}(V) \stackrel{j_{1}^{N}-j_{2}^{N}}{\longrightarrow} \mathcal{F}_{N}(U \cup V) \stackrel{\delta_{N}^{\mathcal{F}}}{\longrightarrow} \ldots \\
\quad \ldots \stackrel{\delta_{i+1}^{\mathcal{F}}}{\longrightarrow} \mathcal{F}_{i}(U \cap V) \stackrel{\left(\iota_{1}^{i}, l_{2}^{i}\right)}{\longrightarrow} \mathcal{F}_{i}(U) \oplus \mathcal{F}_{i}(V) \stackrel{j_{1}^{i}-j_{2}^{i}}{\longrightarrow} \mathcal{F}_{i}(U \cup V) \stackrel{\delta_{i}^{\mathcal{F}}}{\longrightarrow} \ldots \\
\ldots \stackrel{\delta_{1}^{\mathcal{F}}}{\longrightarrow} \mathcal{F}_{0}(U \cap V) \stackrel{\left(\iota_{1}^{0}, l_{2}^{0}\right)}{\longrightarrow} \mathcal{F}_{0}(U) \oplus \mathcal{F}_{0}(V) \stackrel{j_{1}^{0}-j_{2}^{0}}{\longrightarrow} \mathcal{F}_{0}(U \cup V) \longrightarrow 0 .
\end{gathered}
$$

(Note that the only difference to Definition 4.3 in [7] is that our sequence ends to the left with the term $\mathcal{F}_{N}(U \cap V)$ because $\mathcal{F}_{N+1}(U \cup V), \delta_{N+1}^{\mathcal{F}}$, etc. are not defined.) In accordance with Definition 4.3 in [7], a collection of morphisms of precosheaves $f_{i}: \mathcal{F}_{i} \rightarrow \mathcal{G}_{i}, 0 \leq i \leq N$, is called $\delta$-compatible if for every pair of open sets $U, V \in \tau \mathcal{U}$ the following diagram commutes for all $i$ (where $0 \leq i \leq N-1$ in our setting):

$$
\begin{gathered}
\mathcal{F}_{i+1}(U \cup V) \stackrel{\delta_{i+1}^{\mathcal{F}}(U, V)}{\longrightarrow} \mathcal{F}_{i}(U \cap V) \\
f_{i+1}(U \cup V) \downarrow \\
\mathcal{G}_{i+1}(U \cup V) \stackrel{\delta_{i+1}^{\mathcal{G}}(U, V)}{\longrightarrow} \mathcal{G}_{i}(U \cap V) .
\end{gathered}
$$

Next we state our adaption of Proposition 4.4 in [7] to finite sequences of $\delta$ compatible morphisms between precosheaves. (The only change in the proof is that the five lemma is applied to a commutative ladder that ends on the left with the homomorphism $f_{N}\left(V^{j}\right): \mathcal{F}_{N}\left(V^{j}\right) \rightarrow \mathcal{G}_{N}\left(V^{j}\right)$.)

Proposition 4.16. Let $\mathcal{U}$ be an open cover of the topological space B. Let $f_{i}: \mathcal{F}_{i} \rightarrow$ $\mathcal{G}_{i}, 0 \leq i \leq N$, be a finite sequence of $\delta$-compatible morphisms between $\mathcal{U}$ locally constant precosheaves on $B$ that satisfy the $\mathcal{U}$-Mayer-Vietoris property. If the map $f_{i}(U): \mathcal{F}_{i}(U) \rightarrow \mathcal{G}_{i}(U)$ is an isomorphism for every $U \in \mathcal{U}$ and for every $0 \leq i \leq N$, then $f_{i}(B): \mathcal{F}_{i}(B) \rightarrow \mathcal{G}_{i}(B)$ is an isomorphism for all $0 \leq i \leq N$.

Recall that we have a fiber bundle $\pi_{<}: \mathrm{ft}_{<} E \rightarrow B$ with fiber $L_{<}$. Consider also the fiber bundle $\rho: \operatorname{cyl}\left(F_{<}\right) \rightarrow B$ with fiber $\operatorname{cyl}\left(f_{<}\right)$, and note that there are morphisms of fiber bundles $\mathrm{ft}_{<} E \rightarrow \operatorname{cyl}\left(F_{<}\right) \rightarrow E$ restricting to fiberwise maps $L_{<} \rightarrow \operatorname{cyl}\left(f_{<}\right) \rightarrow L$.

Fix a good cover $\mathcal{U}$ of $B$, and set $N=\max \{k, l\}-1$. We apply Proposition 4.16 to the precosheaves $\mathcal{F}_{i}$ and $\mathcal{G}_{i}, 0 \leq i \leq N$, given on open sets $U \in \tau \mathcal{U}$ by $\mathcal{F}_{i}(U)=$ $H_{i}\left(\rho^{-1}(U), \pi_{<}^{-1}(U)\right)$ and $\mathcal{G}_{i}(U)=0$, and the morphisms of precosheaves $f_{i}: \mathcal{F}_{i} \rightarrow \mathcal{G}_{i}$ determined by the unique map $f_{i}(U): H_{i}\left(\rho^{-1}(U), \pi_{<}^{-1}(U)\right) \rightarrow 0$ for open sets $U \in \tau \mathcal{U}$. Note that, by the Eilenberg-Steenrod axioms, the precosheaves $\mathcal{F}_{i}$ are $\mathcal{U}$-locally constant because $\mathcal{U}$ is a good open cover of $B$, and satisfy the 
$\mathcal{U}$-Mayer-Vietoris property with respect to the connecting homomorphisms of the relative form of the Mayer-Vietoris sequence. It is clear that the trivial precosheaves $\mathcal{G}_{i}$ are $\mathcal{U}$-locally constant and satisfy the $\mathcal{U}$-Mayer-Vietoris property with respect to the natural transformations $\delta_{i}^{\mathcal{G}}=0$. Obviously, the morphisms of precosheaves $f_{i}: \mathcal{F}_{i} \rightarrow \mathcal{G}_{i}$ are $\delta$-compatible. Moreover, as every open set $U \in \mathcal{U}$ is contractible, we conclude for every $0 \leq i \leq N(<\max \{k, l\})$ that $f_{i}(U): \mathcal{F}_{i}(U) \rightarrow \mathcal{G}_{i}(U)=0$ is an isomorphism because

$$
\mathcal{F}_{i}(U)=H_{i}\left(\rho^{-1}(U), \pi_{<}^{-1}(U)\right) \cong H_{i}\left(\operatorname{cyl}\left(f_{<}\right), L_{<}\right) \cong \widetilde{H}_{i}\left(\operatorname{cone}\left(f_{<}\right)\right)=0,
$$

where the last equality holds by equation (2). Hence, Proposition 4.16 implies that $\mathcal{F}_{i}(B)=0$ for all $0 \leq i \leq N$, where note that

$$
\mathcal{F}_{i}(B)=H_{i}\left(\operatorname{cyl}\left(F_{<}\right), \mathrm{ft}_{<} E\right) \cong \widetilde{H}_{i}\left(\operatorname{cone}\left(F_{<}\right)\right) .
$$

The following result provides a proof of simple connectivity of truncation cones, at least under a mild hypothesis on the underlying Moore approximation. Simple connectivity of the truncation cone will enable us to invoke the machinery of rational homotopy theory in Sect. 5.

Lemma 4.17. The truncation cone, cone $\left(F_{<}\right)$, is path connected. If the equivariant Moore approximation $f_{<}: L_{<} \rightarrow L$ induces a surjection $f_{<*}: \pi_{1}\left(L_{<}, x_{0}\right) \rightarrow$ $\pi_{1}\left(L, f_{<}\left(x_{0}\right)\right)$ for every basepoint $x_{0} \in L_{<}$, then cone $\left(F_{<}\right)$is simply connected.

Proof. Since $f_{<}: L_{<} \rightarrow L$ is a Moore approximation of positive degree, the induced map $H_{0}\left(L_{<}\right) \rightarrow H_{0}(L)$ is an isomorphism. Hence, cone $\left(f_{<}\right)$, the mapping cone of $f_{<}$, is path connected. As the bundle morphism $F_{<}: \mathrm{ft}_{<} E \rightarrow E$ restricts on fibers to copies of the map $f_{<}$, it follows that cone $\left(F_{<}\right)$is path connected.

It remains to show that $\pi_{1}\left(\operatorname{cone}\left(F_{<}\right)\right.$, pt) is trivial. For this purpose, we note that the bundle morphism $F_{<}: \mathrm{ft}_{<} E \rightarrow E$ can be written as the disjoint union $F_{<}=\bigsqcup_{i} F_{<}^{(i)}$ of bundle morphisms

$$
F_{<}^{(i)}: \mathrm{ft}_{<} E^{(i)} \rightarrow E^{(i)},
$$

where $\mathrm{ft}_{<} E=\bigsqcup_{i} \mathrm{ft}_{<} E^{(i)}$ and $E=\bigsqcup_{i} E^{(i)}$ are decompositions into the path components, and we note that the bundles $\pi_{<}: \mathrm{ft}_{<} E \rightarrow B$ and $\pi: E \rightarrow B$ restrict to bundles $\pi_{<}^{(i)}: \mathrm{ft}_{<} E^{(i)} \rightarrow B$ and $\pi^{(i)}: E^{(i)} \rightarrow B$. (In fact, from every point $x \in E$ there exists a path in $E$ to a point of the form $F_{<}(y)$ for some $y \in \mathrm{ft}_{<} E$ because $F_{<}$restricts over the basepoint $\pi(x) \in B$ to a copy of the map $f_{<}: L_{<} \rightarrow L$, and we can use surjectivity of the induced map $H_{0}\left(L_{<}\right) \rightarrow$ $H_{0}(L)$. Conversely, given two points $y_{0}, y_{1} \in \mathrm{ft}_{<} E$ whose images under $F_{<}$can be connected by a path in $E$, we construct a path between $y_{0}$ and $y_{1}$ in $\mathrm{ft}_{<} E$ as follows. We choose a path $\gamma_{E}:[0,1] \rightarrow E$ from $\gamma_{E}(0)=F_{<}\left(y_{0}\right)$ to $\gamma_{E}(1)=F_{<}\left(y_{1}\right)$. Then, $\gamma_{E}$ is a section of $\pi: E \rightarrow B$ along the path $\gamma=\pi \circ \gamma_{E}:[0,1] \rightarrow B$. In particular we have $F_{<}\left(y_{0}\right) \in \pi^{-1}(\gamma(0))$ and $F_{<}\left(y_{1}\right) \in \pi^{-1}(\gamma(1))$. The pullback of the bundle morphism $F_{<}: \mathrm{ft}_{<} E \rightarrow E$ under $\gamma$ is isomorphic to the bundle morphism $f_{<} \times \operatorname{id}_{[0,1]}: L_{<} \times[0,1] \rightarrow L \times[0,1]$ of trivial bundles over the 
interval $[0,1]$. Under this pullback, the points $y_{0} \in \pi_{<}^{-1}(\gamma(0))$ and $y_{1} \in \pi_{<}^{-1}(\gamma(1))$ correspond to points of the form $\left(y_{0}^{\prime}, 0\right) \in L_{<} \times\{0\}$ and $\left(y_{1}^{\prime}, 1\right) \in L_{<} \times\{1\}$, respectively. Furthermore, the section $\gamma_{E}:[0,1] \rightarrow E$ of $\pi: E \rightarrow B$ pulls back to a section $\gamma_{E}^{\prime}:[0,1] \rightarrow L \times[0,1]$ of the projection $L \times[0,1] \rightarrow[0,1]$ such that $\gamma_{E}^{\prime}(0)=\left(f_{<}\left(y_{0}^{\prime}\right), 0\right) \in L \times\{0\}$ and $\gamma_{E}^{\prime}(1)=\left(f_{<}\left(y_{1}^{\prime}\right), 1\right) \in L \times\{1\}$. Using injectivity of the map $H_{0}\left(L_{<}\right) \rightarrow H_{0}(L)$ induced by $f_{<}$, we conclude that there is a section $\gamma_{<}^{\prime}:[0,1] \rightarrow L_{<} \times[0,1]$ of the projection $L_{<} \times[0,1] \rightarrow[0,1]$ such that $\gamma_{<}^{\prime}(0)=\left(y_{0}^{\prime}, 0\right)$ and $\gamma_{<}^{\prime}(1)=\left(y_{1}^{\prime}, 1\right)$. All in all, the composition of $\gamma_{<}^{\prime}:[0,1] \rightarrow L_{<} \times[0,1]$ with the structure map $L_{<} \times[0,1] \rightarrow \mathrm{ft}_{<} E$ is the desired path in $\mathrm{ft}_{<} E$.) Since cone $\left(F_{<}\right)=\bigvee_{i} \operatorname{cone}\left(F_{<}^{(i)}\right)$, we conclude from the Seifert-van Kampen theorem for a bouquet of spaces (see Example 1.21 in [22, p. 43]) that in order to show that $\pi_{1}\left(\operatorname{cone}\left(F_{<}\right)\right.$, pt) is trivial, it suffices to show that $\pi_{1}\left(\operatorname{cone}\left(F_{<}^{(i)}\right), \mathrm{pt}\right)$ is trivial for every $i$.

Fix an index $i$. The Seifert-van Kampen theorem implies that the fundamental group $\pi_{1}$ (cone $\left.\left(F_{<}^{(i)}\right), \mathrm{pt}\right)$ is trivial when the canonical inclusion $\mathrm{ft}_{<} E^{(i)} \subset$ $\operatorname{cyl}\left(F_{<}^{(i)}\right)$ induces a surjection on fundamental groups. In view of the exactness of the sequence

$$
\pi_{1}\left(\mathrm{ft}_{<} E^{(i)}\right) \rightarrow \pi_{1}\left(\operatorname{cyl}\left(F_{<}^{(i)}\right)\right) \rightarrow \pi_{1}\left(\operatorname{cyl}\left(F_{<}^{(i)}\right), \mathrm{ft}_{<} E^{(i)}\right)
$$

it suffices to show that $\pi_{1}\left(\operatorname{cyl}\left(F_{<}^{(i)}\right), \mathrm{ft}_{<} E^{(i)}\right)$ is trivial. By the discussion in [22, p. 346], this means we have to show that every path $[0,1] \rightarrow \operatorname{cyl}\left(F_{<}^{(i)}\right)$ with endpoints in $\mathrm{ft}_{<} E^{(i)}$ is homotopic rel endpoints to a path $[0,1] \rightarrow \mathrm{ft}_{<} E^{(i)}$.

Let us show that every path $\alpha:[0,1] \rightarrow \operatorname{cyl}\left(F_{<}\right)$with endpoints in $\mathrm{ft}_{<} E \subset$ $\operatorname{cyl}\left(F_{<}\right)$is homotopic rel endpoints to a path $[0,1] \rightarrow \mathrm{ft}_{<} E$. Note that we have a fiber bundle $\rho: \operatorname{cyl}\left(F_{<}\right) \rightarrow B$ with fiber $\operatorname{cyl}\left(f_{<}\right)$, where the canonical inclusion $\mathrm{ft}_{<} E \subset \operatorname{cyl}\left(F_{<}\right)$can fiberwisely be identified with the canonical inclusion $j: L_{<} \rightarrow \operatorname{cyl}\left(f_{<}\right)$. Then, we may consider $\alpha$ as a section of $\rho: \operatorname{cyl}\left(F_{<}\right) \rightarrow B$ along the path $\beta=\rho \circ \alpha:[0,1] \rightarrow B$. The pullback of the bundle $\rho: \operatorname{cyl}\left(F_{<}\right) \rightarrow B$ under $\beta$ can be identified with the trivial bundle $\rho^{\prime}=\operatorname{pr}_{[0,1]}: \operatorname{cyl}\left(f_{<}\right) \times[0,1] \rightarrow[0,1]$ in such a way that the section $\alpha$ of $\rho$ pulls back to a section $\alpha^{\prime}:[0,1] \rightarrow \operatorname{cyl}\left(f_{<}\right) \times[0,1]$ of $\rho^{\prime}$, where $\alpha^{\prime}(0) \in L_{<} \times\{0\}$ and $\alpha^{\prime}(1) \in L_{<} \times\{1\}$. It remains to show that the unique map $\alpha^{\prime \prime}:[0,1] \rightarrow \operatorname{cyl}\left(f_{<}\right)$ satisfying $\alpha^{\prime}(t)=\left(\alpha^{\prime \prime}(t), t\right)$ for all $t \in[0,1]$ is homotopic rel endpoints to a map $[0,1] \rightarrow L_{<}$. In other words, by the discussion in [22, p. 346], we have to show that $\pi_{1}\left(\operatorname{cyl}\left(f_{<}\right), L_{<}, x_{0}\right)=0$ for every basepoint $x_{0} \in L_{<}$. In order to show this, we consider the following portion of the long exact sequence of homotopy groups of the pointed pair $\left(\operatorname{cyl}\left(f_{<}\right), L_{<}, x_{0}\right)$ (omitting the basepoint):

$$
\pi_{1}\left(L_{<}\right) \stackrel{j_{*}}{\longrightarrow} \pi_{1}\left(\operatorname{cyl}\left(f_{<}\right)\right) \rightarrow \pi_{1}\left(\operatorname{cyl}\left(f_{<}\right), L_{<}\right) \rightarrow \pi_{0}\left(L_{<}\right) \stackrel{j_{*}}{\longrightarrow} \pi_{0}\left(\operatorname{cyl}\left(f_{<}\right)\right) .
$$

Note that $j_{*}$ is an isomorphism in degree 0 because $f_{<}$induces an isomorphism $\pi_{0}\left(L_{<}, x\right) \rightarrow \pi_{0}(L, x)$. Thus, the claim follows from the assumption that $f_{<}$ induces a surjection on fundamental groups, i.e., that $j_{*}$ is a surjection in degree 1 . 
Example 4.18. If $L$ is path connected and has abelian fundamental group, then any equivariant Moore approximation $f_{<}: L_{<} \rightarrow L$ to $L$ of degree $\geq 2$ induces automatically a surjection on fundamental groups. In fact, by naturality of the Hurewicz homomorphism we have for any basepoint $x_{0} \in L_{<}$the commutative diagram

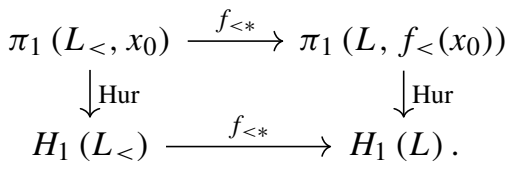

Note that the left vertical map is surjective and the right vertical map is an isomorphism because in degree one, Hurewicz maps are abelianization maps. Hence, the claim follows because $f_{<*}: H_{1}\left(L_{<}\right) \rightarrow H_{1}(L)$ is an isomorphism.

Example 4.19. If $L$ is a path connected cell complex and the fiber bundle $E \rightarrow B$ is trivial, then there exists for any $d \geq 2$ an equivariant Moore approximation $f_{<}: L_{<} \rightarrow L$ to $L$ of degree $d$ which induces a surjection on fundamental groups. Indeed, since the bundle $E \rightarrow B$ is trivial, the structure group $G$ can be chosen to be trivial. Thus, by the results of [28], $L$ admits a cellular Moore approximation $f_{<}: L_{<} \rightarrow L$ of degree $d \geq 2$ in such a way that $f_{<}$restricts to the identity on $(d-1)$-skeleta. In particular, the map induced by $f_{<}$on fundamental groups is surjective.

\section{Rational homotopy theory}

Theorem 4.2 reveals a natural relation between the rational Hurewicz homomorphism of the truncation cone and the condition that in some degree all complementary cup products in the reduced cohomology ring of the truncation cone vanish. In this section we study the homotopy theoretic ramifications of this cohomological vanishing condition (see e.g. Corollary 5.7 and Remark 5.8).

The main purpose of this section is to prove (see Sect. 5.5) the following

Theorem 5.1. Let $n \geq 3$ be an integer. Let $\pi: E^{n-1} \rightarrow B$ be a fiber bundle of closed manifolds with closed manifold fiber $L$ and structure group $G$ such that $B, E$ and $L$ are compatibly oriented. Suppose that B admits a good open cover (this holds whenever $B$ is smooth or at least $P L)$. Let $\bar{p}$ and $\bar{q}$ be complementary perversities, and set $k=c-\bar{p}(c+1)$ and $l=c-\bar{q}(c+1)$, where $c=\operatorname{dim}$ L. Suppose that the fiber $L$ possesses a $G$-equivariant Moore approximation $f_{<}: L_{<} \rightarrow L$ both of degree $k$ and of degree $l$ such that the associated truncation cone cone $\left(F_{<}\right)$is simply connected (compare Lemma 4.17). Furthermore, we suppose that

(1) $n \leq 3 \cdot \max \{k, l\}-1$, and

(2) $\mathcal{O}_{i}(\pi, k, l)=0$ for all $i$.

Then, the rational Hurewicz homomorphism of the associated truncation cone,

$$
\operatorname{Hur}_{n-1 *}: \pi_{n-1}\left(\operatorname{cone}\left(F_{<}\right), \mathrm{pt}\right) \otimes_{\mathbb{Z}} \mathbb{Q} \rightarrow H_{n-1}\left(\operatorname{cone}\left(F_{<}\right)\right),
$$

is surjective. 


\subsection{Minimal Sullivan algebras}

Assuming the ground field to be the rationals, we provide some necessary notation from [16].

By a graded (rational) vector space we mean a collection $V=\left\{V^{p}\right\}_{p \geq 1}$ of rational vector spaces (see [16, p. 40] and [16, p. 138]). We say that $v \in V^{p}$ is an element of $V$ of degree $p$, and write $v \in V$ and $|v|=p$. As in [16, p. 42], we use the notation $V^{\geq p}=\left\{V^{q}\right\}_{q \geq p}, V^{<p}=\left\{V^{q}\right\}_{q<p}$, etc.

As in Example 6 of [16, p. 45], the free graded commutative algebra of $V$ is the quotient algebra $\Lambda V=T V / I$, where $T V$ denotes the tensor algebra of $V$ (see Example 4 in [16, p. 45]), and $I \subset T V$ denotes the ideal generated by all elements of the form $v \otimes w-(-1)^{|v| \cdot|w|} w \otimes v$, where $v, w \in V$. Following [16, p. 140], we also write $\Lambda V^{\geq p}=\Lambda\left(V^{\geq p}\right), \Lambda V^{<p}=\Lambda\left(V^{<p}\right)$, etc.

Note that, as vector spaces, $\Lambda V=\bigoplus_{p=0}^{\infty} \Lambda^{p} V$, where $\Lambda^{p} V$ denotes the linear span of all products $v_{1} \wedge \cdots \wedge v_{p}, v_{1}, \ldots, v_{p} \in V$, of word length $p$ and degree $\left|v_{1}\right|+\cdots+\left|v_{p}\right|$ (see Example 6(vi) in [16, p. 46] as well as [16, p. 140]). We will also write $\Lambda^{\geq p} V=\bigoplus_{q \geq p} \Lambda^{q} V$, and in particular $\Lambda^{+} V=\Lambda^{\geq 1} V$.

Recall from [16, p. 138] that a Sullivan algebra is a commutative cochain algebra of the form $(\Lambda V, d)$ whose differential $d$ satisfies the following nilpotence condition. It is required that there exists an increasing sequence $V(0) \subset \cdots \subset$ $V(k) \subset \ldots$ of graded linear subspaces of $V$ such that $V=\bigcup_{k=0}^{\infty} V(k), d=0$ on $V(0)$, and $d(V(k)) \subset \Lambda V(k-1)$ for all $k>0$. If the differential $d$ of the Sullivan algebra $(\Lambda V, d)$ satisfies in addition im $d \subset \Lambda^{\geq 2} V$, then we call the Sullivan algebra $(\Lambda V, d)$ minimal.

Note that if a Sullivan algebra $(\Lambda V, d)$ is minimal, then the projection

$$
\rho: \Lambda^{+} V=\bigoplus_{q \geq 1} \Lambda^{q} V \rightarrow \Lambda^{1} V=V
$$

induces according to the discussion in $[16$, p. 173] a homomorphism

$$
\zeta: H^{+}(\Lambda V)=\frac{\operatorname{ker}\left(d: \Lambda^{+} V \rightarrow \Lambda^{+} V\right)}{\operatorname{im}\left(d: \Lambda^{+} V \rightarrow \Lambda^{+} V\right)} \rightarrow V, \quad \zeta([z])=\rho(z) .
$$

Lemma 5.2. Fix integers $r, s, t \geq 1$. Suppose that $(\Lambda V, d)$ is a minimal Sullivan algebra whose underlying graded vector space $V$ is of the form $V=\left\{V^{p}\right\}_{p \geq r}$, and whose differential $d$ vanishes on all elements of degree $\leq s$. Furthermore, suppose that all t-complementary products in $H^{+}(\Lambda V)$ vanish, that is, for all classes $\alpha \in H^{i}(\Lambda V), \beta \in H^{t-i}(\Lambda V), 1 \leq i \leq t-1$, we have $\alpha \wedge \beta=0$ in $H^{t}(\Lambda V)$. If $t \leq r+s$, then the associated homomorphism $\zeta: H^{+}(\Lambda V) \rightarrow V$ is injective in degree $t$.

Proof. Suppose that $[z] \in H^{t}(\Lambda V)$ is a class such that $\zeta([z])=0$. That is, $z$ is a cocycle of degree $t$ in $(\Lambda V, d)$ satisfying $\rho(z)=0$. Thus, since $z \in \Lambda^{\geq 2} V$ by definition of $\rho, z$ can be written as a rational linear combination of elements of the form $v_{1} \wedge \cdots \wedge v_{m}$, where $m \geq 2$, and $v_{1}, \ldots, v_{m} \in V$ are nonzero elements such that $\left|v_{1}\right|+\cdots+\left|v_{m}\right|=t$. Since $m \geq 2$, it follows from $\left|v_{1}\right|, \ldots,\left|v_{m}\right| \geq r$ and 
$t \leq r+s$ that $\left|v_{1}\right|, \ldots,\left|v_{m}\right| \leq s$. Consequently, the elements $v_{1}, \ldots, v_{m} \in V \leq s$ are all cocycles in $(\Lambda V, d)$. Hence, $\left[v_{1}\right] \wedge \cdots \wedge\left[v_{m}\right]=0$ in $H^{t}(\Lambda V)$ according to the vanishing assumption on $t$-complementary products in $H^{+}(\Lambda V)$. This shows that $[z]=0$ in $H^{t}(\Lambda V)$.

The following example shows that the assumption $t \leq r+s$ is in general necessary in Lemma 5.2.

Example 5.3. Fix an odd integer $u \geq 3$. Suppose that the only nonzero parts of the graded vector space $V$ are $V^{u}$ with basis $\{x, y\}$, and $V^{2 u-1}$ with basis $\{z\}$. Then, it is easy to check that $(\Lambda V, d)$ is a minimal Sullivan algebra with differential $d$ determined by $d x=d y=0$ and $d z=x y$. (In fact, a sequence $V(0) \subset \cdots \subset$ $V(k) \subset \ldots$ of the required form is given by taking $V(0)$ to be the graded vector space whose only nonzero part is $V(0)^{u}=V^{u}$, and by setting $V(k)=V$ for $k>0$.) By construction, the only non-trivial cohomology groups of $H^{+}(\Lambda V)$ are $H^{u}(\Lambda V)$ (generated by the classes of $x$ and $y$ ), $H^{3 u-1}(\Lambda V)$ (generated by the classes of $x z$ and $y z$ ), and $H^{4 u-1}(\Lambda V)$ (generated by the class of $x y z$ ). Now let $r=u, s=2 u-2$, and $t=r+s+1=3 u-1$. Then, the minimal Sullivan algebra $(\Lambda V, d)$ satisfies all assumptions of Lemma 5.2 except for $t \leq r+s$. Furthermore, the homomorphism $\zeta: H^{+}(\Lambda V) \rightarrow V$ is clearly not injective in degree $t$ because $V^{3 u-1}=0$, whereas $H^{3 u-1}(\Lambda V) \neq 0$.

\subsection{Minimal Sullivan models}

Given any commutative cochain algebra $(A, d)$, a Sullivan model for $(A, d)$ is a Sullivan algebra $(\Lambda V, d)$ together with a quasi-isomorphism $m:(\Lambda V, d) \stackrel{\simeq}{\longrightarrow}$ $(A, d)$. The Sullivan model is called minimal if the corresponding Sullivan algebra $(\Lambda V, d)$ is minimal. It can be shown that any commutative cochain algebra $(A, d)$ with $H^{0}(A) \cong \mathbb{Q}$ possesses a unique minimal Sullivan model (see the corollary to Theorem 14.12 in [16, p. 191]).

Given a commutative cochain algebra $(A, d)$ such that $H^{0}(A) \cong \mathbb{Q}$ and $H^{1}(A)=0$, the following explicit algorithm for constructing the (unique) minimal Sullivan model $m:(\Lambda V, d) \stackrel{\simeq}{\longrightarrow}(A, d)$ is described before Proposition 12.2 in [16, p. 145]. Starting with a morphism $m_{2}:\left(\Lambda V^{2}, 0\right) \rightarrow(A, d)$ such that $H^{2}\left(m_{2}\right): V^{2} \cong H^{2}(A)$, the construction provides inductively for $k=2,3, \ldots$ a vector space $V^{k+1}$, an extension of the (derivational) differential $d$ from $\Lambda V \leq k$ to $\Lambda V^{\leq k+1}=\Lambda V^{\leq k} \otimes \Lambda V^{k+1}$, and an extension of $m_{k}:\left(\Lambda V^{\leq k}, d\right) \rightarrow(A, d)$ to a cochain algebra morphism $m_{k+1}:\left(\Lambda V^{\leq k+1}, d\right) \rightarrow(A, d)$, such that, for all $k=2,3, \ldots, H^{i}\left(m_{k}\right)$ is an isomorphism for $i \leq k$, and $H^{k+1}\left(m_{k}\right)$ is injective. Having constructed $m_{k}:\left(\Lambda V^{\leq k}, d\right) \rightarrow(A, d)$, the next step of the induction is as follows. Choose cocycles $a_{\alpha} \in A^{k+1}$ and $z_{\beta} \in(\Lambda V \leq k)^{k+2}$ such that

$$
H^{k+1}(A)=\operatorname{im} H^{k+1}\left(m_{k}\right) \oplus \bigoplus_{\alpha} \mathbb{Q} \cdot\left[a_{\alpha}\right],
$$

$$
\operatorname{ker} H^{k+2}\left(m_{k}\right)=\bigoplus_{\beta} \mathbb{Q} \cdot\left[z_{\beta}\right] \text {. }
$$


Let $V^{k+1}$ be a vector space with basis $\left\{v_{\alpha}^{\prime}\right\} \cup\left\{v_{\beta}^{\prime \prime}\right\}$ in correspondence with the elements $\left\{a_{\alpha}\right\} \cup\left\{z_{\beta}\right\}$. The (derivational) differential $d$ is extended from $\Lambda V \leq k$ to $\Lambda V^{\leq k+1}=\Lambda V^{\leq k} \otimes \Lambda V^{k+1}$ by setting $d v_{\alpha}^{\prime}=0$ and $d v_{\beta}^{\prime \prime}=z_{\beta}$. Finally, the map $m_{k}:\left(\Lambda V^{\leq k}, d\right) \rightarrow(A, d)$ is extended to a cochain algebra morphism

$$
m_{k+1}:\left(\Lambda V^{\leq k+1}, d\right) \rightarrow(A, d)
$$

via $m_{k+1} v_{\alpha}^{\prime}=a_{\alpha}$ and $m_{k+1} v_{\beta}^{\prime \prime}=b_{\beta}$, where $b_{\beta} \in A^{k+1}$ has been chosen in such a way that $d b_{\beta}=m_{k} z_{\beta}$.

Lemma 5.4. Consider a commutative cochain algebra $(A, d)$ satisfying $H^{0}(A) \cong$ $\mathbb{Q}$ and $H^{1}(A)=0$. Let $r \geq 2$ be the smallest integer such that $H^{r}(A) \neq 0$. If $m:(\Lambda V, d) \stackrel{\simeq}{\longrightarrow}(A, d)$ denotes the (unique) minimal Sullivan model for $(A, d)$, then the underlying graded vector space $V$ is of the form $V=\left\{V^{p}\right\}_{p \geq r}$, and the differential $d$ vanishes on all elements of degree $\leq 2 r-2$.

Proof. The claim $V=\left\{V^{p}\right\}_{p \geq r}$ is part of Proposition 12.2(ii) in [16, p. 145].

In order to show that the differential $d$ vanishes on all elements of degree $\leq$ $2 r-2$, it suffices by the inductive construction of $d$ to show that ker $H^{k+2}\left(m_{k}\right)=0$ for $k=2, \ldots, 2 r-3$. (For $r=2$ there is nothing to show since $d$ vanishes on all elements of degree $\leq 2$ by construction.) Fix $k=2, \ldots, 2 r-3$. If $k<r$, then $V=\left\{V^{p}\right\}_{p \geq r}$ implies that $\left(\Lambda V^{\leq k}\right)^{0}=\mathbb{Q}$ and $\left(\Lambda V^{\leq k}\right)^{p}=0$ for $p>0$. If, however, $k \geq r$, then $V=\left\{V^{p}\right\}_{p \geq r}$ implies that

$$
\left(\Lambda V^{\leq k}\right)^{p}= \begin{cases}\mathbb{Q}, & p=0, \\ 0, & p=1, \ldots, r-1 \\ V^{p}, & p=r, \ldots, k \\ 0, & p=k+1, \ldots, 2 r-1 .\end{cases}
$$

In any case, we see that $\left(\Lambda V^{\leq k}\right)^{k+2}=0$, and, in particular, ker $H^{k+2}\left(m_{k}\right)=0$.

\subsection{Commutative cochain algebras for spaces}

Recall from Section 10 in [16, p. $115 \mathrm{ff}$.] that Sullivan has constructed a contravariant functor $A_{P L}$ from the category of topological spaces and continuous maps to the category of commutative cochain algebras and cochain algebra morphisms. Furthermore, the functor $A_{P L}$ has the important property that for any topological space $X$, the graded algebras $H^{*}(X)$ and $H\left(A_{P L}(X)\right)$ are naturally isomorphic (and can hence be identified).

Given a path connected topological space $X$, we can in particular consider the minimal Sullivan model for $X$, that is, the (unique) minimal Sullivan model $m_{X}:\left(\Lambda V_{X}, d\right) \stackrel{\simeq}{\rightarrow} A_{P L}(X)$ for the commutative cochain algebra $(A, d)=$ $A_{P L}(X)$. Moreover, recall from Sect. 5.1 that we can associate to the minimal Sullivan algebra $\left(\Lambda V_{X}, d\right)$ a homomorphism $\zeta_{X}: H^{+}\left(\Lambda V_{X}\right) \rightarrow V_{X}$.

In Proposition 5.5 below, we characterize the non-vanishing of the rational Hurewicz homomorphism in terms of rational homotopy theory (compare the proof of Proposition 3.14 in [23, p. 250]). 
Proposition 5.5. Let $\left(X, x_{0}\right)$ be a simply connected pointed space, and suppose that $H_{*}(X)$ is of finite type (i.e., the rational vector space $H_{r}(X)$ has finite dimension for all $r \in \mathbb{Z})$. Then for any integer $n \geq 3$ the following statements are equivalent:

(i) The Hurewicz homomorphism

$$
\operatorname{Hur}_{n-1 *}: \pi_{n-1}\left(X, x_{0}\right) \otimes_{\mathbb{Z}} \mathbb{Q} \rightarrow H_{n-1}(X)
$$

is surjective.

(ii) The homomorphism $\zeta_{X}^{n-1}: H^{n-1}\left(\Lambda V_{X}\right) \rightarrow V_{X}^{n-1}$ is injective.

Moreover, if either of the above statements is satisfied for $X$, then all $(n-1)$ complementary cup products in $\widetilde{H}^{*}(X)$ vanish.

Proof. We fix a minimal Sullivan model $m_{X}:\left(\Lambda V_{X}, d\right) \stackrel{\simeq}{\longrightarrow} A_{P L}(X)$ for $X$.

Since $\left(X, x_{0}\right)$ is simply connected and $H_{*}(X)$ is of finite type, the corollary to Theorem 15.11 in $[16$, p. 210] implies that in degree $n-1>1$ the homomorphism $\zeta_{X}^{n-1}: H^{n-1}\left(\Lambda V_{X}\right) \rightarrow V_{X}^{n-1}$ can be identified with the dual

$$
\operatorname{Hur}_{n-1}^{*}: H^{n-1}(X) \rightarrow \operatorname{Hom}_{\mathbb{Z}}\left(\pi_{n-1}(X), \mathbb{Q}\right)
$$

of the Hurewicz map $\operatorname{Hur}_{n-1}: \pi_{n-1}(X) \rightarrow H_{n-1}(X ; \mathbb{Z})$. Since the extension of scalars functor $-\otimes_{\mathbb{Z}} \mathbb{Q}$ is left adjoint to the restriction of scalars functor (along $\mathbb{Z} \subset \mathbb{Q}$ ), the latter homomorphism can furthermore be identified with

$$
\begin{aligned}
& \operatorname{Hom}_{\mathbb{Q}}\left(\operatorname{Hur}_{n-1 *}(-), \mathbb{Q}\right): \operatorname{Hom}_{\mathbb{Q}}\left(H_{n-1}(X) \otimes_{\mathbb{Z}} \mathbb{Q}, \mathbb{Q}\right) \\
& \quad \rightarrow \operatorname{Hom}_{\mathbb{Q}}\left(\pi_{n-1}(X) \otimes_{\mathbb{Z}} \mathbb{Q}, \mathbb{Q}\right) .
\end{aligned}
$$

Thus, the equivalence $(i) \Leftrightarrow$ (ii) follows from the observation that a homomorphism $V \rightarrow W$ of rational vector spaces is surjective if and only if its dual $\operatorname{Hom}_{\mathbb{Q}}(W, \mathbb{Q}) \rightarrow \operatorname{Hom}_{\mathbb{Q}}(V, \mathbb{Q})$ is injective.

Let us suppose that there is a non-vanishing $(n-1)$-complementary cup product in $\widetilde{H}^{*}(X)$, that is, for some $i \in\{1, \ldots, n-2\}$, there exist classes $\alpha \in H^{i}(X)$ and $\beta \in H^{n-1-i}(X)$ such that $0 \neq \alpha \cup \beta \in H^{n-1}(X)$. As $H^{*}\left(A_{P L}(X)\right) \cong H^{*}(X)$ (as graded algebras) and $m_{X}:\left(\Lambda V_{X}, d\right) \stackrel{\simeq}{\longrightarrow} A_{P L}(X)$ is a quasi-isomorphism of commutative differential graded algebras, there exist cocycles $a \in \Lambda V_{X}$ of degree $i$ and $b \in \Lambda V_{X}$ of degree $n-1-i$ such that $0 \neq[a \wedge b] \in H^{n-1}\left(\Lambda V_{X}\right)$. But then $\zeta_{X}^{n-1}$ is not injective because $a \wedge b \in \Lambda^{\geq 2} V_{X}$ implies that $\zeta_{X}([a \wedge b])=\rho(a \wedge b)=0$. Hence, we have shown that all $(n-1)$-complementary cup products in $\widetilde{H}^{*}(X)$ must vanish if $X$ satisfies either of the statements $(i)$ and $(i i)$.

Remark 5.6. In the context of Theorem 4.2 we have exploited statement $(i)$ in our proof that the local duality obstructions vanish. Under the assumption that $X=$ cone $\left(F_{<}\right)$is simply connected (compare Lemma 4.17), Proposition 5.5 provides another proof by means of the minimal Sullivan model of $X$, based on statement (ii). 


\subsection{A rational Hurewicz theorem}

One part of Proposition 5.5 states that in a given degree, surjectivity of the rational Hurewicz homomorphism implies that complementary cup products in the reduced cohomology ring vanish. Our purpose is to prove the converse implication under reasonable hypotheses. This is addressed in Corollary 5.7, where the additional assumption is that the considered degree $n-1$ should not be too large compared to the rational connectedness $r$ of the space $X$. Note that for $n-1=2 r-1$, Corollary 5.7 specializes to a part of the classical rational Hurewicz theorem (for spaces with homology of finite type) because the vanishing condition for complementary cup products is trivially satisfied. This is exactly the implication that is employed in [23] in the context of isolated singularities.

Corollary 5.7. Let $\left(X, x_{0}\right)$ be a simply connected pointed space, and suppose that $H_{*}(X)$ is of finite type (i.e., the rational vector space $H_{r}(X)$ has finite dimension for all $r \in \mathbb{Z}$ ). Let $n \geq 3$ be an integer such that the following assumptions hold:

(1) If $r \geq 2$ denotes the smallest positive integer such that $H^{r}(X) \neq 0$, then $n \leq 3 r-1$.

(2) All $(n-1)$-complementary cup products in $\widetilde{H}^{*}(X)$ vanish, that is, for all $\alpha \in$ $H^{i}(X), \beta \in H^{n-1-i}(X), 1 \leq i \leq n-2$, we have $0=\alpha \cup \beta \in H^{n-1}(X)$.

Then, the rational Hurewicz homomorphism

$$
\operatorname{Hur}_{n-1 *}: \pi_{n-1}\left(X, x_{0}\right) \otimes_{\mathbb{Z}} \mathbb{Q} \rightarrow H_{n-1}(X)
$$

is surjective.

Proof. We consider the commutative cochain algebra $(A, d)=A_{P L}(X)$ and its minimal Sullivan model $m_{X}:\left(\Lambda V_{X}, d\right) \stackrel{\simeq}{\longrightarrow} A_{P L}(X)$. Using that the graded algebras $H^{*}(X)$ and $H^{*}\left(A_{P L}(X)\right)$ are isomorphic, we obtain from Lemma 5.4 that $V_{X}=\left\{V_{X}^{p}\right\}_{p \geq r}$, and that the differential $d$ vanishes on all elements of degree $\leq 2 r-2$, where $r \geq 2$ denotes the smallest positive integer such that $H^{r}(X) \neq 0$. Next, we apply Lemma 5.2 to the minimal Sullivan algebra $\left(\Lambda V_{X}, d\right)$ and the integers $r, s=2 r-2$ and $t=n-1$. Note that $t \leq r+s$ by assumption (1), and all $t$-complementary cup products in $H^{+}\left(\Lambda V_{X}\right)$ vanish by assumption (2) because $m_{X}$ is a quasi-isomorphism. Hence, we conclude that the homomorphism $\zeta_{X}: H^{+}\left(\Lambda V_{X}\right) \rightarrow V_{X}$ is injective in degree $t=n-1$. Finally, the claim that the Hurewicz homomorphism $\operatorname{Hur}_{n-1 *}: \pi_{n-1}\left(X, x_{0}\right) \otimes_{\mathbb{Z}} \mathbb{Q} \rightarrow H_{n-1}(X)$ is surjective follows from implication $(i i) \Rightarrow$ (i) of Proposition 5.5.

Remark 5.8. (formality) In Corollary 5.7, the cohomological vanishing condition in assumption (2) is a "formal shadow" of the statement that the rational Hurewicz homomorphism of $X$ is surjective in degree $n-1$. In fact, let us show that Corollary 5.7 remains valid if we replace assumption (1) by the assumption

(1') The space $X$ is formal (see [16, p. 156]), that is, $A_{P L}(X)$ is connected to $\left(H^{*}(X), 0\right)$ by a chain

$$
A_{P L}(X) \stackrel{\simeq}{\rightarrow}\left(C_{0}, d_{0}\right) \stackrel{\simeq}{\longleftarrow} \ldots \stackrel{\simeq}{\rightarrow}\left(C_{k}, d_{k}\right) \stackrel{\simeq}{\longleftarrow}\left(H^{*}(X), 0\right)
$$

of quasi-isomorphisms of commutative cochain algebras. 
For this purpose, we exploit the well known fact that a minimal Sullivan model $m_{X}:\left(\Lambda V_{X}, d\right) \stackrel{\simeq}{\longrightarrow} A_{P L}(X)$ lifts through a chain of quasi-isomorphisms as in $(1 ')$ to yield a minimal Sullivan model $m:\left(\Lambda V_{X}, d\right) \stackrel{\simeq}{\longrightarrow}\left(H^{*}(X), 0\right)$. We have to prove that the homomorphism $\zeta_{X}: H^{+}\left(\Lambda V_{X}\right) \rightarrow V_{X}$ is injective in degree $n-1$. Suppose that the cocycle $x \in \Lambda V_{X}$ represents a class in $H^{n-1}\left(\Lambda V_{X}\right)$ such that $\zeta_{X}^{n-1}([x])=0$. By definition of the homomorphism $\zeta_{X}$, this means that $\rho(x)=0$ (i.e., $x$ has no linear components). Thus, $x$ can be written as a finite sum of the form

$$
x=\sum_{i} v_{1}^{(i)} \wedge \cdots \wedge v_{l_{i}}^{(i)}
$$

where for every $i$, we have $l_{i} \geq 2$, and $v_{1}^{(i)}, \ldots, v_{l_{i}}^{(i)} \in V_{X}$ are nonzero elements such that $\left|v_{1}^{(i)}\right|+\cdots+\left|v_{l_{i}}^{(i)}\right|=n-1$. By applying the algebra morphism $m$, we obtain

$$
m(x)=\sum_{i} m\left(v_{1}^{(i)}\right) \cup \cdots \cup m\left(v_{l_{i}}^{(i)}\right)=0
$$

because each summand vanishes being an $(n-1)$-complementary cup product in $\widetilde{H}^{*}(X)$ by assumption (2). Therefore, passing to the map on cohomology induced by $m$, we have $H^{n-1}(m)([x])=[m(x)]=0 \in H^{n-1}(X)$. But since $m$ is a quasiisomorphism, it follows that $[x]=0$ in $H^{n-1}\left(\Lambda V_{X}\right)$, which implies that $\zeta_{X}^{n-1}$ is injective. This proves our modified version of Corollary 5.7.

\subsection{Proof of Theorem 5.1}

We wish to apply Corollary 5.7 to the simply connected pointed space $\left(X, x_{0}\right)=$ (cone $\left(F_{<}\right)$, pt). Note that $H_{*}(X)$ is of finite type because the inclusion $E \subset$ $\operatorname{cone}\left(F_{<}\right)$induces a surjective homomorphism $H_{p}(E) \rightarrow H_{p}\left(\operatorname{cone}\left(F_{<}\right)\right)$for all $p \in \mathbb{Z}$ (see properties (a) and (b) of $Q_{\geq} E$ listed in the proof of Theorem 4.2 in Sect. 4.3, where we use the assumption that $B$ admits a good open cover). Since $B$ admits a good open cover, the assumptions (1) and (2) of Theorem 5.1 imply that the conditions (1) and (2) of Corollary 5.7 are satisfied. (Concerning (1), note that the smallest integer $r \geq 2$ such that $H^{r}(X) \neq 0$ satisfies $r \geq \max \{k, l\}$ according to Lemma 4.15. As for condition (2), we apply Lemma 4.1.)

Finally, application of Corollary 5.7 completes the proof of Theorem 5.1.

Remark 5.9. We obtain an alternative proof of Theorem 5.1 by checking assumption (1') of Remark 5.8 instead of assumption (1) of Corollary 5.7. By invoking the obstruction theory developed in [21], we observe that assumption (1') follows in fact from assumption (1) if we impose the additional assumption that the space $X$ satisfies $H^{p}(X)=0$ for $p>n-1$. (Indeed, if we assume (1) as well as $H^{p}(X)=0$ for $p>n-1$, then the commutative graded algebra $H=H^{*}(X)$ satisfies $H^{0}=\mathbb{Q}$, and $H^{p}=0$ for $1 \leq p \leq r-1$ and for $p>3(r-1)+1$. Hence, we can apply Corollary 5.16 in [21] for $H=H^{*}(X), l=r-1$, and $C=A_{P L}(X)$ 
to obtain a chain of quasi-isomorphisms between $C$ and $(H, 0)$ as desired.) But by using the surjective homomorphism $H_{p}(E) \rightarrow H_{p}\left(\operatorname{cone}\left(F_{<}\right)\right)$from the proof of Theorem 5.1, we see that $X=\operatorname{cone}\left(F_{<}\right)$satisfies $H^{p}(X)=0$ for $p>n-1$ because $\operatorname{dim} E=n-1$.

\section{Intersection spaces and the signature}

In Sect. 6.1 we apply the results of the previous sections to study middle perversity intersection spaces of depth one Witt spaces within the framework of Thom-Mather stratified spaces. In particular, given a depth one Witt space $X$ (see Definition 6.2), Theorem 6.3(a) provides our Hurewicz criterion for the existence of a Klimczak completion $\widehat{I X}=I X \cup e^{n}$ of the middle perversity intersection space $I X$. Then, in part (b) of Theorem 6.3, we relate the Hurewicz criterion to the vanishing of the local duality obstructions to Banagl-Chriestenson by showing that they are equivalent if the dimensions of the singular strata are not too big. Moreover, when the dimension $n$ of $X$ is of the form $n=4 d$, Theorem 6.3(c) implies that the signature of the symmetric intersection form $H_{2 d}(\widehat{I X}) \times H_{2 d}(\widehat{I X}) \rightarrow \mathbb{Q}$ equals the signature of the Goresky-MacPherson-Siegel intersection form $I H_{2 d}(X) \times I H_{2 d}(X) \rightarrow \mathbb{Q}$ on middle-perversity intersection homology. Finally, we illustrate our results by a concrete example in Sect. 6.2.

\subsection{Depth one Witt spaces}

Before focusing on depth one Witt spaces and their middle perversity intersection spaces (see Theorem 6.3), we discuss more generally intersection spaces of stratified pseudomanifold of depth 1 along the lines of [7].

First of all, an $n$-dimensional two strata pseudomanifold is a pair $(X, \Sigma)$ consisting of a locally compact, second countable Hausdorff space $X$ and a closed connected subspace $\Sigma$ such that $\Sigma \subset X$ is equipped with a Thom-Mather $C^{\infty}$ stratification of $X$ as follows. The regular stratum $X \backslash \Sigma$ is a smooth $n$-manifold that is dense in $X$, and the singular stratum $\Sigma$ is a smooth manifold whose codimension in $X$ is at least 2 . Moreover, $\Sigma$ is required to be equipped with so-called Thom-Mather control data (for details, see Section 8 of [7]).

Suppose that $(X, \Sigma)$ is an $n$-dimensional two strata pseudomanifold with nonempty singular stratum $\Sigma$. Then, as explained at the beginning of Section 9 in [7], the Thom-Mather control data of $\Sigma \subset X$ can be used to construct an open neighborhood $U$ of $\Sigma$ in $X$ and a smooth (locally trivial) fiber bundle $\pi: E \rightarrow B$ with the following properties. The complement $M=X \backslash U$ is a smooth $n$-manifold with boundary $\partial M=E$, and there exists a homeomorphism from the closure $U \cup \partial M$ of $U$ in $X$ to the homotopy pushout $D E$ of $B \stackrel{\pi}{\longleftarrow} E \stackrel{\operatorname{id}_{E}}{\longrightarrow} E$ that restricts to diffeomorphisms $\Sigma \cong B$ and $(U \cup \partial M) \backslash \Sigma \cong D E \backslash B=E \times(0,1]$ extending $\partial M=E \cong E \times\{1\}$. We assume that the data $(U, \pi: E \rightarrow B)$ have been fixed, and call $U$ a regular neighborhood, and the fiber bundle $\pi: E \rightarrow B$ the link bundle of the singular stratum $\Sigma=B$. The (non-empty) fiber $L$ of $\pi$ is called the link of the singular stratum $\Sigma=B$. 
More generally (see Definition 8.3 in [7]), an $n$-dimensional stratified pseudomanifold of depth 1 is a tuple $\left(X, \Sigma_{1}, \ldots, \Sigma_{r}\right)$, where the $\Sigma_{i}$ are mutually disjoint subspaces of $X$ such that for every $i=1, \ldots, r$, the pair $\left(X \backslash \bigcup_{j \neq i} \Sigma_{j}, \Sigma_{i}\right)$ is a two strata pseudomanifold whose singular stratum $\Sigma_{i}$ has regular neighborhood $U^{(i)}$, and link bundle $\pi^{(i)}: E^{(i)} \rightarrow B^{(i)}$ having link $L^{(i)}$. The stratified depth 1 pseudomanifold $\left(X, \Sigma_{1}, \ldots, \Sigma_{r}\right)$ is called oriented if the top stratum $X \backslash \bigcup_{i} \Sigma_{i}$ is equipped with an orientation.

Consider an $n$-dimensional stratified pseudomanifold $X=\left(X, \Sigma_{1}, \ldots, \Sigma_{r}\right)$ of depth 1 . Given a perversity $\bar{p}$, we proceed to explain the construction of the $\bar{p}$-intersection space $I^{\bar{p}} X$, which will depend on the choice of equivariant Moore approximations of the link bundles of the singular strata. We denote by $c_{i}$ the dimension of the link $L^{(i)}$ of the singular stratum $\Sigma_{i}$, and set $k_{i}=c_{i}-\bar{p}\left(c_{i}+1\right)$, which is a positive integer. For every $i$ we assume that the link $L^{(i)}$ possesses for some choice of structure group $G^{(i)}$ of the link bundle $\pi^{(i)}$ a $G^{(i)}$-equivariant Moore approximation of degree $k_{i}$, say

$$
f_{<k_{i}}^{(i)}: L_{<k_{i}}^{(i)} \rightarrow L^{(i)}
$$

As explained in the beginning of Sect. 4, the Moore approximations $f_{<k_{i}}^{(i)}$ induce fiberwise truncations

$$
F_{<k_{i}}^{(i)}: \mathrm{ft}_{<k_{i}} E^{(i)} \rightarrow E^{(i)}
$$

where $\mathrm{ft}_{<k_{i}} E^{(i)}$ is the total space of the fiber bundle $\pi_{<k_{i}}^{(i)}: \mathrm{ft}_{<k_{i}} E^{(i)} \rightarrow$ $B^{(i)}$ obtained by replacing the fiber $L^{(i)}$ of $\pi^{(i)}$ with the fiber $L_{<k_{i}}^{(i)}$, and $F_{<k_{i}}^{(i)}: \mathrm{ft}_{<k_{i}} E^{(i)} \rightarrow E^{(i)}$ is induced by $f_{<k_{i}}^{(i)}: L_{<k_{i}}^{(i)} \rightarrow L^{(i)}$ in such a way that $\pi^{(i)} \circ F_{<k_{i}}^{(i)}=\pi_{<k_{i}}^{(i)}$. Observe that if we choose the regular neighborhoods $U^{(i)}$ sufficiently small so that $U^{(i)} \cap U^{(j)}=\emptyset$ for $i \neq j$, then $M=X \backslash \bigcup_{i} U^{(i)}$ is a smooth manifold with boundary $\partial M=\bigsqcup_{i} E^{(i)}$.

Definition 6.1. (compare Definition 9.1 in [7]) The perversity $\bar{p}$ intersection space $I^{\bar{p}} X$ of the depth 1 pseudomanifold $X=\left(X, \Sigma_{1}, \ldots, \Sigma_{r}\right)$ is defined as the homotopy cofiber of the composition

$$
\bigsqcup_{i} \mathrm{ft}_{<k_{i}} E^{(i)} \stackrel{\bigsqcup_{i} F_{<k_{i}}^{(i)}}{\longrightarrow} \bigsqcup_{i} E^{(i)}=\partial M \stackrel{\text { incl }}{\longrightarrow} M .
$$

In other words, $I^{\bar{p}} X=\operatorname{cone}\left(\bigsqcup_{i} F_{<k_{i}}^{(i)}\right) \cup_{\partial M} M$ is the homotopy pushout of

$$
\mathrm{pt} \longleftarrow \bigsqcup_{i} \mathrm{ft}_{<k_{i}} E^{(i)} \stackrel{\tau}{\longrightarrow} M
$$

From now on, we are concerned with Witt spaces, which are an important class of stratified pseudomanifolds defined by Siegel [25]. 
Definition 6.2. (see Definition 8.3 in [7]) An oriented depth 1 stratified pseudomanifold $\left(X, \Sigma_{1}, \ldots, \Sigma_{r}\right)$ is called Witt space if the following condition is satisfied. For each $1 \leq i \leq r$ such that the dimension $c_{i}$ of the link $L^{(i)}$ of the singular stratum $\Sigma_{i}$ is even, we have $H_{\frac{c_{i}}{2}}\left(L^{(i)}\right)=0$.

We specialize to the case of the lower middle perversity $\bar{p}=\bar{m}$ and the upper middle perversity $\bar{q}=\bar{n}$. Consider a compact depth one Witt space $\left(X, \Sigma_{1}, \ldots, \Sigma_{r}\right)$. Suppose that $f_{<}^{(i)}: L_{<}^{(i)} \rightarrow L^{(i)}$ is a $G^{(i)}$-equivariant Moore approximation of degree $k_{i}=c_{i}-\bar{n}\left(c_{i}+1\right)=\left\lfloor\frac{1}{2}\left(c_{i}+1\right)\right\rfloor$. Then, it follows from the homology vanishing condition of Definition 6.2 that $f_{<}^{(i)}$ is also a $G^{(i)}$-equivariant Moore approximation of $L^{(i)}$ of complementary degree $l_{i}=c_{i}-\bar{m}\left(c_{i}+1\right)=$ $\left\lceil\frac{1}{2}\left(c_{i}+1\right)\right\rceil$. Thus, the resulting intersection spaces $I^{\bar{m}} X$ and $I^{\bar{n}} X$ of Definition 6.1 can be chosen to be equal, $I^{\bar{m}} X=I^{\bar{n}} X$.

Let us state the main result of this section.

Theorem 6.3. Let $\left(X, \Sigma_{1}, \ldots, \Sigma_{r}\right)$ be a compact depth one Witt space of dimension $n \geq 3$. Assume that for every $i=1, \ldots, r$ the link $L^{(i)}$ of the singular stratum $\Sigma_{i}$ admits an equivariant Moore approximation $f_{<}^{(i)}: L_{<}^{(i)} \rightarrow L^{(i)}$ of degree $k_{i}=$ $\left\lfloor\frac{1}{2}\left(c_{i}+1\right)\right\rfloor$ (and thus, also one of degree $\left.l_{i}=\left\lceil\frac{1}{2}\left(c_{i}+1\right)\right\rceil\right)$. Let $F_{<}^{(i)}: \mathrm{ft}_{<} E^{(i)} \rightarrow$ $E^{(i)}$ denote the fiberwise truncation of the link bundle $\pi^{(i)}: E^{(i)} \rightarrow B^{(i)}$. Then, for the resulting middle perversity intersection space

$$
I X=\operatorname{cone}\left(\bigsqcup_{i} F_{<}^{(i)}\right) \cup_{\partial M} M
$$

of Definition 6.1, the following statements hold:

(a) If the rational Hurewicz homomorphism

$$
\operatorname{Hur}_{n-1 *}: \pi_{n-1}\left(\operatorname{cone}\left(F_{<}^{(i)}\right), \mathrm{pt}\right) \otimes_{\mathbb{Z}} \mathbb{Q} \rightarrow H_{n-1}\left(\operatorname{cone}\left(F_{<}^{(i)}\right)\right)
$$

is surjective for every $i$, then the middle perversity intersection space IX admits a completion $\widehat{I X}=I X \cup e^{n}$ to a rational Poincaré duality space by attaching a single $n$-cell.

(b) Fix an index $i \in\{1, \ldots, r\}$. If the rational Hurewicz homomorphism in part (a) is surjective, then the local duality obstructions $\mathcal{O}_{*}\left(\pi^{(i)}, k_{i}, l_{i}\right)$ vanish. The converse implication holds at least when the truncation cone, cone $\left(F_{<}^{(i)}\right)$, is simply connected (see Lemma 4.17), and

$$
\operatorname{dim} B^{(i)}< \begin{cases}\left(\operatorname{dim} L^{(i)}+1\right) / 2, & \text { if } \operatorname{dim} L^{(i)} \text { is odd }, \\ \left(\operatorname{dim} L^{(i)}+4\right) / 2, & \text { if } \operatorname{dim} L^{(i)} \text { is even } .\end{cases}
$$

(c) Suppose that the dimension of $X$ is of the form $n=4 d$. Furthermore, suppose that the rational Hurewicz homomorphism in part (a) is surjective for every $i$, and let $\widehat{I X}=I X \cup e^{n}$ be a completion to a Poincaré duality space as provided by part $(a)$. Then, the Witt element $w_{H I} \in W(\mathbb{Q})$ induced by the symmetric intersection form $H_{2 d}(\widehat{I X}) \times H_{2 d}(\widehat{I X}) \rightarrow \mathbb{Q}$ of the Poincaré duality 
space $\widehat{I X}$ equals the Witt element $w_{I H} \in W(\mathbb{Q})$ induced by the GoreskyMacPherson-Siegel intersection form $I_{2 d} H_{2}(X) \times I H_{2 d}(X) \rightarrow \mathbb{Q}$ on middleperversity intersection homology (see Section I.4.1 in [25]). In particular, the two intersection forms have equal signatures.

Proof. (a). Fix $i \in\{1, \ldots, r\}$. In the following, we let $f^{(i)}: Y^{(i)} \rightarrow Z^{(i)}$ denote the map $F_{<}^{(i)}: \mathrm{ft}_{<} E^{(i)} \rightarrow E^{(i)}$, and set $X^{(i)}=\operatorname{cone}\left(f^{(i)}\right)$. By the same argument as in the first half of the proof of Theorem 4.2 (see Sect. 4.3), we can show that the map $f^{(i)}: Y^{(i)} \rightarrow Z^{(i)}$ and its mapping cone $X^{(i)}$ satisfy all the assumptions of Proposition 4.9. Then, it follows that the assumptions of Proposition 4.9 are also satisfied for the map $f: Y \rightarrow Z$ given by $\bigsqcup_{i} f^{(i)}: \bigsqcup_{i} Y^{(i)} \rightarrow \bigsqcup_{i} Z^{(i)}$, and its mapping cone $X=\bigvee_{i} X^{(i)}$. In the following, we will construct a map $\phi: S^{n-1} \rightarrow$ $X$ such that statement $(i i)$ of Proposition 4.9 holds for the map $f: Y \rightarrow Z$ and its mapping cone $X$. Then, $(\operatorname{cone}(\phi), Z)$ is an oriented Poincaré duality pair by the implication $(i i) \Rightarrow(i)$ of Proposition 4.9. Finally, the claim of statement $(a)$ follows by applying the gluing principle for Poincaré duality pairs (see Theorem 3.3 in [23]) to glue the oriented Poincaré duality pairs (cone $(\phi), Z)$ and $(M, \partial M)$ along their common oriented boundary $Z=\partial M$.

Since the rational Hurewicz homomorphism

$$
\operatorname{Hur}_{n-1 *}: \pi_{n-1}\left(X^{(i)}, \mathrm{pt}\right) \otimes_{\mathbb{Z}} \mathbb{Q} \rightarrow H_{n-1}\left(X^{(i)}\right)
$$

is surjective by assumption, the image of the orientation class $\left[Z^{(i)}\right] \in H_{n-1}\left(Z^{(i)}\right)$ of the Poincaré space $Z^{(i)}=E^{(i)}$ under the map $H_{n-1}\left(Z^{(i)}\right) \rightarrow H_{n-1}\left(X^{(i)}\right)$ induced by the inclusion $Z^{(i)} \subset X^{(i)}$ lies in the image of $\operatorname{Hur}_{n-1 *}$. Thus, as a part of the implication (ii) $\Rightarrow$ (i) of Theorem 4.2, there exists a map $\phi^{(i)}:\left(S^{n-1}, s_{0}\right) \rightarrow\left(X^{(i)}, \mathrm{pt}\right)$ such that the orientation class $\left[Z^{(i)}\right] \in H_{n-1}\left(Z^{(i)}\right)$ of the Poincare space $Z^{(i)}=E^{(i)}$ lies in the image of the connecting homomorphism $\partial_{n}: H_{n}\left(\operatorname{cone}\left(\phi^{(i)}\right), Z^{(i)}\right) \rightarrow H_{n-1}\left(Z^{(i)}\right)$. Pick basepoints $y_{i} \in Y^{(i)}$, and let $\bar{Z} \subset X$ denote the mapping cone of the restriction $f \mid:\left\{y_{1}, \ldots, y_{r}\right\} \rightarrow \bigsqcup_{i} Z^{(i)}=Z$. For $i=1, \ldots, r$ we choose pairwise disjoint embeddings $\iota_{i}: D^{n-1} \rightarrow S^{n-1} \backslash\left\{s_{0}\right\}$. After choosing identifications $S^{n-1} /\left(S^{n-1} \backslash \iota_{i}\left(D^{n-1} \backslash \partial D^{n-1}\right)\right)=S^{n-1}$ of pointed spaces, the quotient map $S^{n-1} \rightarrow S^{n-1} /\left(S^{n-1} \backslash \iota_{i}\left(D^{n-1} \backslash \partial D^{n-1}\right)\right)$ can be considered as a map $q^{(i)}:\left(S^{n-1}, s_{0}\right) \rightarrow\left(S^{n-1}, s_{0}\right)$ of degree 1 . Let

$$
\phi:\left(S^{n-1}, s_{0}\right) \rightarrow X=\bigvee_{i} X^{(i)}
$$

denote the map uniquely determined by $r^{(i)} \circ \phi=\phi^{(i)} \circ q^{(i)}$ for all $i=1, \ldots, r$, where $r^{(i)}: X=\bigvee_{i} X^{(i)} \rightarrow X^{(i)}$ is the collapse map given by $\left.r^{(i)}\right|_{X^{(i)}}=\mathrm{id}_{X^{(i)}}$ and $\left.r^{(i)}\right|_{X^{(j)}}=$ pt $\in X^{(i)}$ for $j \neq i$. Let $R^{(i)}$ : $\operatorname{cone}(\phi) \rightarrow \operatorname{cone}\left(\phi^{(i)}\right)$ denote the canonical map of homotopy cofibers induced by the pair $\left(q^{(i)}, r^{(i)}\right)$. (That is, writing cone $(\phi)=\left(S^{n-1} \times[0,1] \sqcup \mathrm{pt} \sqcup X\right) / \sim_{\phi}$ and cone $\left(\phi^{(i)}\right)=\left(S^{n-1} \times\right.$ $\left.[0,1] \sqcup \mathrm{pt} \sqcup X^{(i)}\right) / \sim_{\phi^{(i)}}$, we have $R^{(i)}(\mathrm{pt})=(\mathrm{pt}), R^{(i)}([(s, t)])=\left[\left(q^{(i)}(s), t\right)\right]$ for $(s, t) \in S^{n-1} \times[0,1]$, and $R^{(i)}([x])=\left[r^{(i)}(x)\right]$ for $x \in X$.) Note that $R^{(i)}$ restricts to the collapse map $S^{(i)}: \bar{Z} \rightarrow \bar{Z}^{(i)}:=\operatorname{cone}\left(\left.f\right|_{\left\{y_{i}\right\}}\right)$ that extends $\operatorname{id}_{\bar{Z}^{(i)}}$ by 
the constant map to the cone point of $\bar{Z}^{(i)}$. Writing $\rho_{i}=R_{*}^{(i)}$ and $\sigma_{i}=S_{*}^{(i)}$ for the induced maps on homology, we have the commutative diagram

$$
\begin{array}{ccc}
H_{n}(\operatorname{cone}(\phi), Z) \stackrel{\partial_{n}}{\longrightarrow} & H_{n-1}(Z) \\
\cong \downarrow \text { incl }_{*} & \cong \downarrow \text { incl }_{*} \\
H_{n}(\operatorname{cone}(\phi), \bar{Z}) \stackrel{\partial_{n}}{\longrightarrow} & H_{n-1}(\bar{Z}) \\
\cong \downarrow\left(\rho_{1}, \ldots, \rho_{r}\right) & \cong \downarrow\left(\sigma_{1}, \ldots, \sigma_{r}\right) \\
\bigoplus_{i} H_{n}\left(\operatorname{cone}\left(\phi^{(i)}\right), \bar{Z}^{(i)}\right) \stackrel{\oplus_{i} \partial_{n}}{\longrightarrow} \bigoplus_{i} H_{n-1}\left(\bar{Z}^{(i)}\right) \\
\cong \uparrow \oplus_{i} \text { incl }_{*} & \cong \uparrow \oplus_{i} \text { incl }_{*} \\
\bigoplus_{i} H_{n}\left(\operatorname{cone}\left(\phi^{(i)}\right), Z^{(i)}\right) \stackrel{\oplus_{i} \partial_{n}}{\longrightarrow} \bigoplus_{i} H_{n-1}\left(Z^{(i)}\right),
\end{array}
$$

in which all vertical arrows are isomorphisms by the Eilenberg-Steenrod axioms because $n-1>0$. Note that the orientation class $[Z] \in H_{n-1}(Z)$ of the Poincaré space $Z=E$ corresponds to the tuple $\left(\left[Z^{(1)}\right], \ldots,\left[Z^{(r)}\right]\right) \in \bigoplus_{i} H_{n-1}\left(Z^{(i)}\right)$ via the vertical arrows on the right hand side. (In fact, using the inclusions $\iota_{i}: Z^{(i)} \rightarrow Z$, $i \in\{1, \ldots, r\}$, we note that the map

$\Sigma: \bigoplus_{i} H_{n-1}\left(Z^{(i)}\right) \rightarrow H_{n-1}(Z), \quad \Sigma\left(z_{1}, \ldots, z_{r}\right)=\iota_{1 *}\left(z_{1}\right)+\cdots+\iota_{r *}\left(z_{r}\right)$,

satisfies $\Sigma\left(\left[Z^{(1)}\right], \ldots,\left[Z^{(r)}\right]\right)=[Z]$, and fits into the commutative diagram

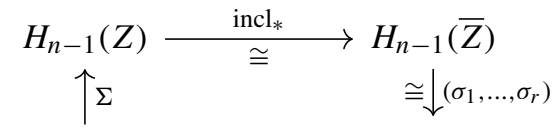

$$
\begin{aligned}
& \bigoplus_{i} H_{n-1}\left(Z^{(i)}\right) \stackrel{\oplus_{i} \text { incl }_{*}}{\cong} \bigoplus_{i} H_{n-1}\left(\bar{Z}^{(i)}\right),
\end{aligned}
$$

whose commutativity can be checked element-wise by using that the composition

$$
Z^{(i)} \stackrel{\iota_{i}}{\rightarrow} Z \stackrel{\text { incl }}{\longrightarrow} \bar{Z} \stackrel{S^{(j)}}{\longrightarrow} \bar{Z}^{(j)}
$$

is the inclusion $Z^{(i)} \rightarrow \bar{Z}^{(i)}$ for $i=j$, and the constant map to the cone point pt $\in \bar{Z}^{(j)}$ for $i \neq j$.) Hence, it follows from diagram (6) that the orientation class $[Z] \in H_{n-1}(Z)$ of the Poincaré space $Z=E$ lies in the image of the connecting homomorphism $\partial_{n}: H_{n}(\operatorname{cone}(\phi), Z) \rightarrow H_{n-1}(Z)$, which is the desired statement (ii) in Proposition 4.9.

(b). In view of Theorem 4.2, the claim follows directly from Theorem 5.1.

(c). We generalize the proof of Corollary 3.10 in [23] to depth one Witt spaces with non-isolated singularities as follows. In view of Corollary 10.2 in [7], the Witt element $w_{I H} \in W(\mathbb{Q})$ equals the Witt element $\widehat{w}_{I H} \in W(\mathbb{Q})$ induced by the Goresky-MacPherson-Siegel intersection form $\Phi_{I H}: I H_{2 d}(\widehat{M}) \times I H_{2 d}(\widehat{M}) \rightarrow \mathbb{Q}$ of the Witt space $\widehat{M}=\operatorname{cone}(\partial M \stackrel{\text { incl }}{\longrightarrow} M)$ with one isolated singularity. Let $d_{M}: H_{2 d}(M) \rightarrow H^{2 d}(M, \partial M)$ be the Poincaré-Lefschetz duality isomorphism of 
$(M, \partial M)$, and let $\alpha: H_{2 d}(M) \rightarrow H_{2 d}(M, \partial M)$ be the map induced by the inclusion of pairs $(M, \emptyset) \rightarrow(M, \partial M)$. As explained in the proof of Theorem 2.28 in [3, p. 137], we have explicitly $I H_{2 d}(\widehat{M})=\operatorname{im} \alpha$ and $\Phi_{I H}(v, w)=d_{M}\left(v^{\prime}\right)(w)$ for any $v^{\prime} \in H_{2 d}(M)$ with $\alpha\left(v^{\prime}\right)=v$. Thus, $\widehat{w}_{I H} \in W(\mathbb{Q})$ is just the Witt element of the Poincaré duality pair $(M, \partial M)$ of dimension $n=4 d$ with orientation class $[M]$ as considered in the context of Lemma 3.4 in [23]. Next, note that $\widehat{I X}$ is the gluing of $(M, \partial M)$ along the oriented boundary $\partial M$ with the oriented Poincaré duality pair (cone $\left.\left(\bigsqcup_{i} F_{<}^{(i)}\right) \cup e^{n}, \partial M\right)$ obtained from the construction of part (a). Thus, we can apply Novikov additivity of Witt elements under gluing of Poincaré duality pairs (see Lemma 3.4 in [23]) to obtain $w_{H I}=\widehat{w}_{I H}-w_{\Sigma}$ in $W(\mathbb{Q})$, where $w_{\Sigma} \in W(\mathbb{Q})$ denotes the Witt element of the oriented Poincaré duality pair $\left(X^{\phi}, Z\right)=\left(\operatorname{cone}\left(\bigsqcup_{i} F_{<}^{(i)}\right) \cup e^{n}, \partial M\right)$. To conclude the claim that $w_{H I}=w_{I H}$ in $W(\mathbb{Q})$, it remains to show that $w_{\Sigma}=0$. For this purpose, recall that $w_{\Sigma} \in W(\mathbb{Q})$ can be represented by a pairing on the image of the canonical map $H_{2 d}\left(X^{\phi}\right) \rightarrow H_{2 d}\left(X^{\phi}, Z\right)$. But the inclusion $Z \rightarrow X^{\phi}$ induces a surjection $H_{2 d}(Z) \rightarrow H_{2 d}\left(X^{\phi}\right)$ because the inclusion $Z \rightarrow X$ induces a surjection $H_{2 d}(Z) \rightarrow H_{2 d}(X)$ by condition (1) of Proposition 4.9, and the inclusion $X \rightarrow X^{\phi}$ induces an isomorphism $H_{2 d}(X) \cong H_{2 d}\left(X^{\phi}\right)$ by Remark 4.11 because $2 d \neq n-1$. Hence, $H_{2 d}\left(X^{\phi}\right) \rightarrow H_{2 d}\left(X^{\phi}, Z\right)$ is the zero map, and the claim follows.

Remark 6.4. (two strata Witt spaces) If we apply Theorem 6.3 for a two strata Witt space $(X, \Sigma)$, then the statements of the parts (a), (b), and (c) specialize to Theorem 2.1, Theorem 2.2, and Theorem 2.3, respectively. The proof of part (a) simplifies for two strata Witt spaces in such a way that we can deduce Theorem 2.1 more directly from Theorem 4.2. Namely, we apply the implication $(i i) \Rightarrow(i)$ of Theorem 4.2 to the link bundle $\pi: E^{n-1} \rightarrow B$ of the singular stratum $\Sigma=B$ of $X$, and to the given equivariant Moore approximation $f_{<}: L<\rightarrow L$ of degree $k=\left\lfloor\frac{1}{2}(\operatorname{dim} L+1)\right\rfloor$ (and thus, also of degree $\left.l=\left\lceil\frac{1}{2}(\operatorname{dim} L+1)\right\rceil\right)$ of the link $L$. As a result, we obtain a completion of the associated truncation cone cone $\left(F_{<}\right)$to an oriented Poincaré duality pair $\left(\operatorname{cone}\left(F_{<}\right) \cup e^{n}, E\right)$ of dimension $n$ whose orientation class extends the orientation $[E] \in H_{n-1}(E)$ of the boundary. Finally, we apply the gluing principle for Poincaré duality pairs (see Theorem 3.3 in [23]) to the oriented Poincaré duality pairs (cone $\left.\left(F_{<}\right) \cup e^{n}, E\right)$ and $(M, \partial M)$ with common oriented boundary $E=\partial M$ to obtain a Klimczak completion $\widehat{I X}=\left(\operatorname{cone}\left(F_{<}\right) \cup e^{n}\right) \cup_{\partial M}$ $M=I X \cup e^{n}$ of the middle perversity intersection space $I X=\operatorname{cone}\left(F_{<}\right) \cup_{\partial M} M$.

Remark 6.5. In general, we do not know how the choice of the attaching map affects the homotopy type of the the completion $\widehat{I X}=I X \cup e^{n}$ constructed in Theorem 6.3(a). However, if the intersection space $I X$ is simply connected, then the following theorem of Stasheff applied for $W=I X$ implies that the rational homotopy type of $Z=\widehat{I X}$ is determined by $I X$.

Theorem 6.6. (Theorem 1 in [27]) Let $H$ be a Poincaré duality algebra of top dimension $n$ and $H^{1}=0$. Let $W$ be a simply connected rational space with $H(W) \cong$ $H$ except $H^{n}(W)=0$. If $Z=W \cup e^{n}$ with $H(Z) \cong H$, then the rational homotopy type of $Z$ is determined by $W$. 
Remark 6.7. Passing from Witt elements to signatures in our proof of Theorem 6.3(c), we recover the statement of Theorem 11.3 in [7], saying that the signature of the intersection form on $H_{2 d}(\widehat{I X})=H_{2 d}(I X)$ equals the so-called Novikov signature of the manifold-with-boundary $(M, \partial M)$. Note that the latter is shown in [7] for the intersection space of a closed oriented two strata Witt space under the assumption that the local duality obstructions of the link bundle vanish. The proof given in [7] is involved, and requires to construct an intersection form of the intersection space $I X$ that is symmetric. As in our proof of Theorem 6.3(c), one also exploits surjectivity of the map $H_{2 d}(Z) \rightarrow H_{2 d}(X)$, namely in diagram (11.2) in [7], where the map takes the form $C_{\geq k *}: H_{m}(\partial M) \rightarrow H_{m}\left(Q_{\geq k} E\right)$.

\subsection{An example}

To illustrate a non-trivial case in which Theorem 6.3 applies, we discuss a class of examples of Witt spaces having as singular strata a finite number of circles with twisted link bundles. As input data for our construction, we employ commutative diagrams of the form

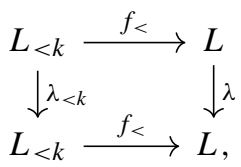

where

- $L$ is a closed oriented smooth manifold of dimension $c>0$,

- $f_{<}: L_{<} \rightarrow L$ is a Moore approximation of $L$ of degree $\left\lfloor\frac{1}{2}(c+1)\right\rfloor$,

- $\lambda: L \rightarrow L$ is an orientation preserving diffeomorphism,

- $\lambda_{<}: L_{<} \rightarrow L_{<}$is a homeomorphism,

such that

- $H_{\frac{c}{2}}(L)=0$ when $c$ is even,

- $f_{<}$induces a surjection $\pi_{1}\left(L_{<}, x_{0}\right) \rightarrow \pi_{1}\left(L, f_{<}\left(x_{0}\right)\right)$ for every $x \in L_{<}$, and

- $\lambda^{N}=\mathrm{id}_{L}$ and $\lambda_{L}^{N}=\mathrm{id}_{L}$ for some integer $N>0$.

Given a commutative diagram as above, we consider the mapping torus

$$
E=(L \times[0,1]) /(x, 0) \sim(\lambda(x), 1)
$$

as the total space of a smooth fiber bundle $\pi: E \rightarrow S^{1}$ with fiber $L$ and structure group $G=\mathbb{Z} / N \mathbb{Z}$ acting on $L$ via $i+N \mathbb{Z} \mapsto \lambda^{i}$. The bundle $\pi$ is flat in the sense that we can choose $G$-valued transition functions that are locally constant. Recall that the integers $k=\left\lfloor\frac{1}{2}(c+1)\right\rfloor$ and $l=\left\lceil\frac{1}{2}(c+1)\right\rceil$ are associated to the uppermiddle perversity $\bar{n}$ and the lower-middle perversity $\bar{m}$, which are complementary. By the properties of the above diagram, the map $f_{<}: L_{<} \rightarrow L$ is a $G$-equivariant Moore approximation both of degree $k$ and of degree $l$. It can be shown that the local duality obstructions $\mathcal{O}_{*}(\pi, k, l)$ vanish. (In fact, we can follow the proof of Theorem 7.1 in [7], which applies literally when we replace the universal cover 
$\widetilde{B} \rightarrow B$ by the $N$-sheeted cover $S^{1} \rightarrow S^{1}$ having the finite group $\pi_{1}=\mathbb{Z} / N$ as its group of deck transformations.) Let $F_{<}: \mathrm{ft}_{<} E \rightarrow E$ denote the fiberwise truncation induced by the $G$-equivariant Moore approximation $f_{<}: L_{<} \rightarrow L$. The truncation cone of $f_{<}$, cone $\left(F_{<}\right)$, is simply connected by Lemma 4.17 . Hence, Theorem 6.3(b) implies that the rational Hurewicz homomorphism

$$
\operatorname{Hur}_{n-1 *}: \pi_{n-1}\left(\operatorname{cone}\left(F_{<}\right), \mathrm{pt}\right) \otimes_{\mathbb{Z}} \mathbb{Q} \rightarrow H_{n-1}\left(\operatorname{cone}\left(F_{<}\right)\right)
$$

is surjective.

We choose a suitable finite disjoint union $\bigsqcup_{i} E^{(i)}$ of mapping tori $E^{(i)}$ coming from the previous construction that can be realized as the boundary of a compact oriented smooth manifold $M$ of dimension $n=c+2$. For instance, when $c=6$, then we can use a single mapping torus because $\Omega_{7}^{S O}=0$. Denoting the homotopy pushout of $S^{1} \stackrel{\pi}{\longleftarrow} E^{(i)} \stackrel{\mathrm{id}_{E^{(i)}}}{\longrightarrow} E^{(i)}$ by $D E^{(i)}$, we thus obtain a compact depth one Witt space $X=M \cup_{\partial M} \bigsqcup_{i} D E^{(i)}$ of dimension $n \geq 3$. Its middle perversity intersection space is given by

$$
I X=\operatorname{cone}\left(\bigsqcup_{i} F_{<}^{(i)}\right) \cup_{\partial M} M,
$$

and according to Theorem 6.3(a), there is a completion $\widehat{I X}=I X \cup e^{n}$ to a rational Poincaré duality space by attaching a single $n$-cell. By Theorem 6.3(c), the signature of the Poincaré duality space $\widehat{I X}$ agrees with the signature of the GoreskyMacPherson-Siegel intersection form on middle-perversity intersection homology of $X$.

In conclusion, let us discuss an example for a commutative diagram as above.

Example 6.8. Consider a closed smooth manifold $K$ of dimension $c \geq 3$ such that $H_{\frac{c}{2}}(K)=0$ when $c$ is even. We fix a $c$-dimensional triangulation $K^{0} \subset \cdots \subset K^{c}$ of $K$. Set $k=\left\lfloor\frac{1}{2}(c+1)\right\rfloor$. The $(k-1)$-skeleton $K^{k-1}$ can be extended to a $k$-dimensional $C W$ complex $K_{<}$in such a way that there exists a Moore approximation $g_{<}: K_{<} \rightarrow K^{k}$ of degree $k$ which restricts to the identity map on $K^{k-1}$. (When $K$ is simply connected and $k \geq 3$, this can be achieved by using Proposition 1.6 of [3]. More generally, note that Proposition 1.3 in [28] can be applied whenever $k \geq 2$, and without assuming $K$ to be simply connected.) The connected sum $L=K \sharp K$ is a closed smooth $c$-manifold which admits an orientation preserving diffeomorphism $\lambda: L \rightarrow L$ interchanging the two summands in such a way that $\lambda^{2}=\mathrm{id}_{L}$. We may equip $L$ with a $c$-dimensional $\mathrm{CW}$ structure $L^{0} \subset \cdots \subset L^{c}$ in such a way that $L^{c-1}=K^{c-1} \vee_{x_{0}} K^{c-1}$ for some basepoint $x_{0} \in K^{0}$, and such that $\lambda$ restricts to the homeomorphism $L^{c-1} \rightarrow L^{c-1}$ that interchanges the two copies of $L^{c-1}$. (To achieve this, we fix a $c$-simplex $\Delta_{c}$ of $K$, choose an embedded closed unit ball $B^{c}$ in the interior of $\Delta_{c}$, and delete the interior $U^{c}$ of $B^{c}$. Then, we form the connected sum $L=K \sharp K$ by gluing two copies of $K \backslash U^{c}$ via the identity map on $\partial B^{c}$. In order to find the desired CW structure on $L$, we modify $B^{c}$ by moving one point of $\partial B^{c}$ to a 0 -simplex $\left\{x_{0}\right\} \subset \partial \Delta_{c}$. Then, we see that $L^{c-1}=K^{c-1} \vee_{x_{0}} K^{c-1}$, and the $c$-cells of $L$ are given by the $c$-simplices of the two copies of $K$ that are different from $\Delta_{c}$, plus one new $c$-cell whose attaching 
map arises from gluing the two copies of $\Delta_{c}$ along the modified $\partial B^{c}$.) We define a degree $k$ Moore approximation $f_{<}: L_{<} \rightarrow L$ by taking the composition

$$
K_{<} \vee_{x_{0}} K_{<} \stackrel{g_{<} \vee g_{<}}{\longrightarrow} K^{k} \vee_{x_{0}} K^{k}=L^{k} \hookrightarrow L=K \sharp K .
$$

Finally, we define $\lambda_{<}: L_{<} \rightarrow L_{<}$to be the homeomorphism that interchanges the two copies of $K_{<}$in the bouquet $L_{<}=K_{<} \vee_{x_{0}} K_{<}$. Then, the desired diagram commutes by construction.

Acknowledgements We would like to thank the reviewer for providing invaluable comments that helped improving the paper. We are grateful to Markus Banagl, Osamu Saeki, and Timo Essig for several discussions. Also, we thank Manuel Amann for a brief correspondence leading to Example 5.3. The author has been supported by JSPS KAKENHI Grant Numbers JP17H06128 and JP18F18752. At the time this work was completed, the author was a JSPS International Research Fellow (Postdoctoral Fellowships for Research in Japan (Standard)).

Open Access This article is licensed under a Creative Commons Attribution 4.0 International License, which permits use, sharing, adaptation, distribution and reproduction in any medium or format, as long as you give appropriate credit to the original author(s) and the source, provide a link to the Creative Commons licence, and indicate if changes were made. The images or other third party material in this article are included in the article's Creative Commons licence, unless indicated otherwise in a credit line to the material. If material is not included in the article's Creative Commons licence and your intended use is not permitted by statutory regulation or exceeds the permitted use, you will need to obtain permission directly from the copyright holder. To view a copy of this licence, visit http://creativecommons.org/ licenses/by/4.0/.

\section{References}

[1] Agustín, M., Fernández de Bobadilla, J.: Intersection space constructible complexes. arXiv: 1804.06185

[2] Banagl, M.: Singular Spaces and Generalized Poincaré Complexes. Electron. Res. Announc. Math. Sci. 16, 63-73 (2009)

[3] Banagl, M.: Intersection Spaces, Spatial Homology Truncation, and String Theory. Lecture Notes in Mathematics, no. 1997, Springer, Berlin (2010)

[4] Banagl, M.: First cases of intersection spaces in stratification depth 2. J. Singul. 5, 57-84 (2012)

[5] Banagl, M.: Foliated stratified spaces and a De Rham complex describing intersection space cohomology. J. Differ. Geom. 104(1), 1-58 (2016)

[6] Banagl, M., Budur, N., Maxim, L.: Intersection spaces, perverse sheaves and type IIB string theory. Adv. Theor. Math. Phys. 18, 363-399 (2014)

[7] Banagl, M., Chriestenson, B.: Intersection spaces, equivariant moore approximation and the signature. J. Singul. 16, 141-179 (2017)

[8] Banagl, M., Hunsicker, E.: Hodge theory for intersection space cohomology. Geom. Topol. 23, 2165-2225 (2019)

[9] Banagl, M., Maxim, L.: Deformation of singularities and the homology of intersection spaces. J. Topol. Anal. 4, 413-448 (2012)

[10] Banagl, M., Maxim, L.: Intersection spaces and hypersurface singularities. J. Singul. 5, 48-56 (2012) 
[11] Browder, W.: Surgery on Simply-Connected Manifolds. Springer, New York (1972)

[12] Cheeger, J.: On the spectral geometry of spaces with cone-like singularities. Proc. Natl. Acad. Sci. USA 76, 2103-2106 (1979)

[13] Cheeger, J.: On the Hodge theory of Riemannian pseudomanifolds. Proc. Symp. Pure Math. 36, 91-146 (1980)

[14] Cheeger, J.: Spectral geometry of singular Riemannian spaces. J. Differ. Geom. 18, 575-657 (1983)

[15] Essig, J.T.: Intersection space cohomology of three-strata pseudomanifolds. J. Topol. Anal. (2019). https://doi.org/10.1142/S1793525320500120

[16] Félix, Y., Halperin, S., Thomas, J.-C.: Rational Homotopy Theory. Springer, Berlin (2001)

[17] Geske, C.: Algebraic intersection spaces. J. Topol. Anal. (2019). https://doi.org/10. 1142/S1793525319500778

[18] Goresky, M., MacPherson, R.D.: Intersection homology theory. Topology 19, 135-162 (1980)

[19] Goresky, M., MacPherson, R.D.: Intersection homology II. Invent. Math. 71, 77-129 (1983)

[20] Goresky, M., MacPherson, R.: Problems and bibliography on intersection homology. Prog. Math. 50, 221-229 (1984)

[21] Halperin, S., Stasheff, J.: Obstructions to homotopy equivalences. Adv. Math. 32, 233279 (1979)

[22] Hatcher, A.: Algebraic Topology. Cambridge University Press, Cambridge (2002)

[23] Klimczak, M.: Poincaré duality for spaces with isolated singularities. Manuscr. Math. 153, 231-262 (2017)

[24] Maxim, L.: Intersection spaces, perverse sheaves and string theory. J. Singul. 15, 118125 (2016)

[25] Siegel, P.H.: Witt spaces: a geometric cycle theory for KO-homology at odd primes. Am. J. Math. 105(5), 1067-1105 (1983)

[26] Spiegel, M.: K-Theory of Intersection Spaces, PhD thesis, Heidelberg University (2013)

[27] Stasheff, J.: Rational Poincaré duality spaces, Ill. J. Math. 27, 104-109 (1983)

[28] Wrazidlo, D.J.: Induced Maps Between Intersection Spaces, Master's thesis, Heidelberg University (2013). urn:nbn:de:bsz:16-heidok-232706

Publisher's Note Springer Nature remains neutral with regard to jurisdictional claims in published maps and institutional affiliations. 